\title{
SENSIBILIDADE DE CULTIVARES DE SOJA (Glycine max) AOS HERBICIDAS DICLOSULAM E FLUMETSULAM ${ }^{1,2}$
}

\author{
Célio R. F. Leite ${ }^{3}$,José C. V. Almeida ${ }^{4}$ e Cássio E. C. Prete ${ }^{4}$
}

\section{RESUMO}

O objetivo deste trabalho foi verificar, no campo, no município de Londrina-PR, a resposta de 12 cultivares de soja em relação aos herbicidas diclosulam e flumetsulam aplicados e incorporados em Latossolo Roxo distrófico $(80,84$ $\%$ de argila, pH 5,4 e matéria orgânica 3,07 \%). As cultivares foram: 'FT-Guaira', 'Embrapa-48', 'Ocepar-14', 'BR-16', 'Embrapa-4', 'Ocepar-13', 'BR-36', 'FT10-Princesa', 'FT-2000', 'FTAbyara', 'BR-37' e 'RS7- Jacuí'. O diclosulam foi utilizado nas doses de $35 \mathrm{~g} / \mathrm{ha}$ (normal) e $70 \mathrm{~g} / \mathrm{ha}$ (dobrada); e o flumetsulam nas doses de $120 \mathrm{~g} / \mathrm{ha}$ (normal) e $240 \mathrm{~g} / \mathrm{ha}$ (dobrada); também foi utilizada a mistura de diclosulam + flumetsulam $(20+100 \mathrm{~g} / \mathrm{ha})$; e imazaquin à $150 \mathrm{~g} / \mathrm{ha}$. Nas doses normais utilizadas dos dois herbicidas não foi possível distinguir nenhuma cultivar suscetível. Em dose dobrada do diclosulam os rendimentos das cultivares 'FT-Guaira', 'Embrapa
4' e 'BR-37' foram reduzidos em 20,9\%, 11,8 \% e $11,0 \%$ respectivamente, em relação à testemunha. O flumetsulam à $240 \mathrm{~g} / \mathrm{ha}$ e sua mistura com diclosulam também reduziu o rendimento destas cultivares, mais o da 'Ocepar14'. A maioria das cultivares teve o estande reduzido, nas doses dobradas dos dois herbicidas, mas também sem significância. Somente o estande da cultivar 'Embrapa-48' não foi afetado, ao contrário da 'RS-7 Jacuí'. A altura de plantas foi menor em relação à testemunha no tratamento com flumetsulam à $240 \mathrm{~g} / \mathrm{ha}$. Na maioria das cultivares o peso da biomassa seca de nódulos foi menor nas doses dobradas, e em algumas também o peso por unidade de nódulos. A biomassa seca da parte aérea e da raiz não foram reduzidas.

Palavras chave: Sulfonanilidas, tolerância, injúria.

\section{ABSTRACT}

\section{Soybean (Glycine max) cultivars sensibility to the herbicides diclosulam and flumetsulam}

The objective of the present paper was to check the behaviour of 12 different soybean cultivars in relation to the tolerance to the herbicides flumetsulam and diclosulam applied on a soil classified as Latossolo Roxo distrófico $(80,87 \%$ of clay, $\mathrm{pH}$ of 5,4 and 3,07 of organic matter) in Londrina - PR. The cultivars were: 'FTGuaira', 'Embrapa-48', 'Ocepar-14', 'BR-16',
'Embrapa-4', 'Ocepar-13', 'BR-36', 'FT 10Princesa', 'FT-2000', 'FT-Abyara', 'BR-37' and 'RS 7-Jacuí'. Diclosulam was used in the rates of $35 \mathrm{~g} / \mathrm{ha}$ (normal rate) and $70 \mathrm{~g} / \mathrm{ha}$ (double rate) and flumetsulam in the rates of $120 \mathrm{~g}$ and $240 \mathrm{~g} / \mathrm{ha}$ (normal and double rate respectively). It was also used a mix of diclosulam and flumetsulam $(20+$ $100 \mathrm{~g} / \mathrm{ha}$ ) and imazaquin at $150 \mathrm{~g} / \mathrm{ha}$.

\footnotetext{
${ }^{1}$ Recebido para publicação em 20/07/98 e na forma revisada 21/01/2000.

2 Parte da dissertação do primeiro autor apresentada à UEL - Universidade Estadual de Londrina para obtenção do título de Mestre em Agronomia.

${ }^{3}$ Eng $^{\circ}$. Agr $^{\circ}$ - SPRAY DROP, Londrina/PR, CEP: 86072-280.

${ }^{4}$ Prof. Adjunto, Universidade Estadual de Londrina, Londrina/PR, CEP: 86051-970.
} 
At the normal rates of both herbicides it was not poss to find any difference in suscetibily of the cultivars.

At double rates of diclosulam the yields of 'FT-Guaira', 'Embrapa-4' and 'BR-37' were reduced in $20,9 \%, 11,8 \%$ and $11,0 \%$, respectively, in relation to the check. Flumetsulam at $240 \mathrm{~g} / \mathrm{ha}$ and its mix with diclosulam also reduced the yields of these cultivars, plus 'Ocepar-14'. The major of the cultivars showed a reduced stand at

\section{INTRODUÇ̃̃O}

Ocasionalmente têm sido notados sinais visuais de injúria em lavouras de soja tratada com flumetsulam, cujos sintomas são descritos como intensa clorose foliar. No entanto, ainda não foi identificada nenhuma cultivar com suscetibilidade diferenciada ao herbicida, bem como são desconhecidos efeitos negativos sobre o rendimento da lavoura, uma vez que os sintomas desaparecem com o passar do tempo.

Sabe-se que a resistência de cultivares de uma mesma espécie de cultura, inclusive a soja, podem responder diferencialmente ao estresse químico causado pelos herbicidas, em razão de diferenças genotípicas (Wax et al., 1976; Beltrão et al., 1983; Gazziero \& Menoso,1984; Cunha et al., 1984.a,b; Friesen et al., 1986; Martin et al., 1987; Buzzell \& Hamill, 1988; Weese et al., 1989; Eberlein et al., 1989; Sander \& Barrett, 1989; Wixon \& Shaw, 1991; Newsom \& Shaw, 1992; Dayan et. al., 1997 ).

Um exemplo bastante conhecido refere-se a extrema sensibilidade de algumas cultivares de soja ao herbicida metribuzin, controlada por um gene recessivo Rps-b hm, enquanto uma marcada tolerância foi associada com a presença do gene Rps1-K Hm (Edwards, et al., 1976). A maioria das cultivares carrega o alelo $\mathrm{Hm}$, porém hm está presente em algumas cultivares (Kilen, 1986 citado por Buzzel \& Hamill,1988). A resposta diferencial de cultivares de soja ao metribuzin aparentemente resulta do aumento do metabolismo em cultivares tolerantes comparado aos cultivares suscetíveis (Falb \& Smith, 1984). O principal the double rates of the both herbicides, but without significance. Only the stand of 'Embrapa48' was not affected, contrary to 'RS 7-Jacuí'. The plant height was reduced by flumetsulam at 240 $\mathrm{g} / \mathrm{ha}$. In all cultivars the nodule dry biomass was reduced in double rates, and in some of them the weight per unit of nodule. The dry biomass of aerial port and roots werw nor reduced.

Key words : Sulfonanilides, tolerance, injury.

metabólito da cultivar 'Coker 102' e 'Semmes', sensíveis ao metribuzin foi [ 6-tert-butyl-astriazin-3,5(2H,4H) dione], enquanto na cultivar tolerante 'Bragg' o principal metabólito foi a conjugação com glicose (Smith \& Wilkinson, 1974 citados por Mangeot et al., 1979). Pereira et al. (1988) em experimentos de campo no cerrado brasileiro verificaram que a cultivar 'Doko' foi a mais tolerante aos herbicidas metribuzin e cyanazine, enquanto 'Engopa-302' e 'FT-11' foram as mais sensíveis. Gazziero \& Menoso (1984) também estudaram o comportamento de 24 cultivares de soja recomendadas para o Estado do Paraná frente ao herbicida metribuzin, e verificaram que a cultivar 'FT-1' foi muito sensível. Cultivares de soja também têm demonstrado respostas diferenciadas de tolerância ao imazaquin e chlorimuron (Newsom \& Shaw, 1992); ao AC 263,222, uma imidazolinona, (Wixon \& Shaw, 1991); ao sulfentrazone (Dayan, 1997).

No Brasil existem atualmente dezenas de cultivares de soja que, devido ao descompasso de seus lançamentos e dos lançamentos dos herbicidas no mercado, não foram investigadas em detalhes com relação a suas resistências a determinados herbicidas. Portanto, sem essas informações, cultivares e herbicidas são utilizados, na maioria das vezes, sem a preocupação da suscetibilidade do material. Assim, um potencial problema de diminuição de nosso rendimento existe e pode passar desapercebido. Uma forma rápida e prática de selecionar cultivares comerciais tolerantes a herbicidas, é submetê-las à ação direta desses 
herbicidas à campo, e avaliar possíveis alterações de suas características morfológicas ou de produção (Martin et al., 1987; Pereira et al., 1988; Weese et al., 1989).

Os ingredientes ativos flumetsulam $(\mathrm{N}-$ (2,6-difluorofenil)-5-metil(1,2,4) triazolo -[1,5a]pirimidina-2-sulfonamida) e diclosulam ( $\mathrm{N}-(2,6-$ diclorofenil)-5-ethoxi-7-fluoro(1,2,4) triazolo [1,5-c] pirimidina-2-sulfonamida ) são os nomes comuns de herbicidas do grupo Triazolopirimidina sulfonanilida para o controle de plantas daninhas dicotiledôneas e seletivos para a cultura da soja (Kleschick et al., 1990, 1992; Bronhara \& Mengarda, 1993.a,b ). Hodges et al., (1990) relatam que a seletividade do flumetsulam é resultado da detoxificação metabólica. Estes autores também relatam que o principal metabólito em soja, de um importante representante do grupo, o herbicida $\mathrm{N}-(2,6-$ dichlorophenyl)- 5,7-dimethyl-1,2,4-triazolo [1,5a]pyrimidine-2-sulfonamide, é a hidroxilação seguida de conjugação com glicose. Podem ser utilizados em pré-plantio e incorporados ou em pré-emergência da cultura. Estes produtos são absorvidos pelas raízes e folhas e se translocam via xilema e floema até os pontos de crescimento onde atuam inibindo a enzima Aceto Lactato Sintetase (ALS), envolvida na biossíntese dos aminoácidos valina, leucina e isoleucina (Gerwick et al., 1990; Kleschick et al., 1990). Experimentos de campo realizados com o flumetsulam indicaram injúrias na soja, a qual se apresentou com clorose foliar e redução do crescimento (Magalhães \& Barros, 1993; Guimarães \& Valente, 1995); mas não foi o que ocorreu em experimentos realizados por Rocha (1993), Bronhara \& Mengarda (1993), nos quais não registraram sinais de injúria na soja.

Em decorrência da falta de informações detalhadas sobre a tolerância das principais cultivares de soja cultivadas atualmente no sul do Brasil em relação aos herbicidas flumetsulam e diclosulam, o objetivo do presente trabalho foi: avaliar o comportamento das principais cultivares de soja comercialmente utilizadas no sul do Brasil, em relação à utilização destes dois herbicidas.

\section{MATERIAL E MÉTODOS}

O presente experimento foi conduzido no período agrícola de 1995/96 na fazenda Escola da Universidade Estadual de Londrina, no município de Londrina, PR, na latitude de $23^{\circ} 18^{\prime} \mathrm{S}$ e longitude de $51^{\circ} 10^{\prime} \mathrm{W}$, com clima classificado como Cfa na escala de Köeppen. O solo no local do ensaio é identificado como Latossolo Roxo distrófico, textura argilosa, contendo 80,84\% de argila, silte 13,64 , pH 5,4 e 3,07\% de matéria orgânica na profundidade de 0,0 a $0,2 \mathrm{~m}$.

$\mathrm{O}$ delineamento experimental utilizado foi o de blocos completos casualizados, com parcelas subdivididas, com 84 tratamentos e 4 repetições. Sendo as cultivares (12) as parcelas, medindo $3,0 \times 42,0$ metros $\left(126,0 \mathrm{~m}^{2}\right)$ e os tratamentos com herbicidas (6), mais a testemunha não tratada (1) as sub-parcelas, medindo $3,0 \times 6,0$ metros $\left(18,0 \mathrm{~m}^{2}\right)$, resultando num total de 336 subparcelas. As cultivares foram semeadas espaçados de 1,0 m uma da outra em cada bloco.

Foram selecionadas 12 cultivares: 'FTGuaira', 'Embrapa-48', 'Ocepar-14', (precoces), 'BR-16', 'Embrapa-4', 'Ocepar-13', 'BR-36' (semi-precoces), 'FT10-Princesa', 'FT-2000', 'FT- Abyara' , 'BR-37' e 'RS7- Jacuí' (ciclo médio).

Os tratamentos herbicidas foram diclosulam (N-(2,6-diclorofenil)-5-ethoxi-7fluoro(1,2,4)triazolo-[1,5-c] pirimidina-2sulfonamida) e flumetsulam (N-(2,6difluorofenil)-5-metil(1,2,4) triazolo -[1,5a]pirimidina-2-sulfonamida) nas doses de 35 e $120 \mathrm{~g} / \mathrm{ha}$ (1X a dose recomendada) e $2 \mathrm{X}$ a dose recomendada (70 e 240 g.i.a./ha) respectivamente; diclosulam + flumetsulam nas doses de 20 + $100 \mathrm{~g} / \mathrm{ha}$; e imazaquin (ácido 2-(4,5 dihidro - 4 metil - 4 (1 - metiletil) - 5 - oxo - $1 \mathrm{H}$ - imidazol - 2 - ilo) - 3 -quinolinacarboxílico) na dose de $150 \mathrm{~g} / \mathrm{ha}$; além da testemunha não tratada. 
A aplicação dos tratamentos em préplantio (PP) foi realizada no dia 08/11/95 (seis dias antes do plantio da soja) no horário das 16:30 às 20:30 horas, em condições de preparo do solo muito bom. A incorporação dos herbicidas foi realizada no dia seguinte (8:00 as 8:15 hs) através do uso de uma grade niveladora de discos, numa única passada, numa profundidade de aproximadamente $10 \mathrm{~cm}$.

Para aplicação dos tratamentos foi utilizado um pulverizador costal propelido a $\mathrm{CO}_{2}$, com pressão constante de $280 \mathrm{Kpa}$, equipado com barra de compensação, contendo 4 bicos Teejet do tipo XR 110:02. A aplicação foi feita a uma altura de $35 \mathrm{~cm}$ do solo e a uma velocidade de 1 $\mathrm{m} / \mathrm{segundo}$, atingindo uma faixa aplicada de 2,0 $\mathrm{m}$ de largura, centralizada longitudinalmente nas sub-parcelas e propiciando um volume de 200 litros de calda por hectare. No momento da aplicação as condições climáticas eram de céu claro, ventos praticamente ausentes $(2 \mathrm{~km} / \mathrm{h})$, temperatura de $26^{\circ} \mathrm{C}$, umidade relativa do ar de $62 \%$ e o solo encontrava-se com pouca umidade. Nos primeiros 7 dias após á aplicação houve precipitações de $30 \mathrm{~mm}$, com mais $30 \mathrm{~mm}$ depois de outros 7 dias; totalizando $252 \mathrm{~mm}$ em 60 dias. A testemunha foi mantida no limpo através de capinas periódicas.

A semeadura das cultivares foram realizadas individualmente no sistema convencional no dia 14/11/95, com uma densidade de aproximadamente 25 sementes por metro no espaçamento de $0,41 \mathrm{~m}$ entre linhas, compondo 7 linhas por parcela. No momento da semeadura o solo encontrava-se em boas condições de preparo e úmido, aparentemente suficiente para a germinação, em função de boas precipitações. As plantas daninhas não controladas pelos herbicidas foram controladas manualmente.

Para avaliar o diferencial de tolerância das cultivares foram medidos, além de observações visuais de injúria, estande, altura de plantas, inserção da primeira vagem, diâmetro de caule, número, peso total e por unidade de nódulos, peso seco da parte aérea e da raiz, rendimento de grãos e peso de 100 sementes.
Aos 17 dias após a emergência da soja , foram contadas o número de plantas em 2 metros das três linhas centrais em cada sub-parcela, respeitando-se 2 metros de cada cabeceira, e para análise foi considerado o número de plantas por metro. Entre 50 e 65 dias, a medida que 20,0 \% de plantas de cada cultivar atingia o florescimento (R2), eram colhidas ao acaso 10 plantas de cinco linhas centrais da área útil $(2,0 \times 4,0 \mathrm{~m})$ de cada sub-parcela. As plantas foram arrancadas manualmente, depois de o solo ao redor do caule ter sido afofado através de uma pá reta. Esta operação foi facilitada pela extrema umidade do solo, dada a freqüência de chuvas naquele período, condição esta imprescindível para um adequado recolhimento de raízes e nódulos. As amostras foram armazenadas em um abrigo para as devidas medições. Foram, então, seccionadas em parte aérea e raiz. Na parte aérea, mediu-se, altura de plantas e biomassa seca, depois de secadas a $70^{\circ} \mathrm{C}$, por 24 horas, em estufa com circulação de ar, e pesadas em balança de precisão, com 3 dígitos depois da vírgula. Das raízes foram primeiramente retirados os nódulos, manualmente, sobre uma peneira de malha fina, com água corrente, para lavagem tanto das raízes quanto dos nódulos. Estes, após secagem a sombra, foram contados. Posteriormente foram colocados para secar a $70^{\circ} \mathrm{C}$, por 24 horas, em estufa com circulação de ar, e em seguida pesados numa balança analítica, com 4 dígitos depois da vírgula. As raízes também foram secas e pesadas da mesma forma que a parte aérea, para a determinação da biomassa seca. Para a determinação do rendimento de grãos, quando da maturação fisiológica (ao redor de 15,0\% de umidade) foram colhidas plantas de 3 linhas de 3 metros da região central de cada sub-parcela, trilhando-as mecanicamente. Posteriormente foram feitas as pesagens, determinando-se a umidade e corrigindo-a para $11,0 \%$, com transformação para $\mathrm{kg} / \mathrm{ha}$. Nestas plantas foram também determinados, na região do colo, o diâmetro $(\mathrm{mm})$ do caule, através de um paquímetro, e altura de inserção da primeira vagem. Foi determinado o peso de 100 sementes, 
em balança de precisão com 3 dígitos depois da vírgula. Além destas foram realizadas observações visuais, baseada na escala de avaliação visual de ensaios de herbicidas da Sociedade Brasileira da Ciência das Plantas Daninhas - SBCPD (1995), qual seja, a aplicação de notas de 0 a $100 \%$ de injúria na cultura da soja.

Os dados colhidos foram submetidos a análise de variância, com exceções das variáveis diâmetro de caule e número de nódulos cujos dados não se adequaram aos seus pressupostos. Foi utilizado o Teste de Tukey ao nível de 5\% de probabilidade, para comparação entre as médias. Algumas variáveis foram transformadas por apresentarem heterogeneidade de variâncias, verificada através da análise de resíduos. Adotouse a metodologia sugerida por Box et al. (1978) para obter a transformação adequada.

\section{RESULTADOS E DISCUSSÃO}

O estande (Tabela 1), na média geral, foi reduzido em uma planta por metro, em relação à testemunha. Nas doses dobradas o efeito foi mais acentuado em grande parte das cultivares. As cultivares 'BR-36', 'BR-16', 'RS-7 Jacuí', 'FTGuaira', 'Ocepar-13', 'Embrapa 4', 'FT-Abyara' e 'BR-37' tiveram os estandes reduzidos de uma a quatro plantas, com flumetsulam à $240 \mathrm{~g} / \mathrm{ha}$, com destaques para a primeira e a última, em mais de três plantas por metro. Com diclosulam à $70 \mathrm{~g} / \mathrm{ha}$ os efeitos foram menores, e somente nas cultivares 'RS-7 Jacuí', 'BR-37', ' BR-36', 'FT-Abyara' e FT-2000, sendo mais expressivo nas duas primeiras. Com a mistura dos dois herbicidas todas estas já mencionadas, à exceção desta última, também tiveram os estandes diminuídos, evidenciando-se a 'Ocepar 13' com quatro plantas a menos. Com imazaquin, apenas 'FT-Guaíra', 'FT-2000' e Embrapa-48 não foram afetadas. Esta última, tão somente, não foi afetado por nenhum dos tratamentos com herbicida em relação ao estande. Por outro lado o estande da 'RS-7 Jacuí' foi menor em todos os tratamentos com herbicida.

A altura de plantas (Tabela 2) foi aumentada na maioria dos tratamentos com herbicida, exceto com flumetsulam à $240 \mathrm{~g} / \mathrm{ha}$. E as duas cultivares mais afetadas foram 'Embrapa4' e 'Ocepar-14' , mas não foi significativo. É importante ressaltar que houve uma correspondência entre a maior altura de plantas e produção, estímulo atribuído, talvez, ao efeito do herbicida.

A inserção da primeira vagem (Tabela 3) foi diminuída, não significativamente, apenas na cultivar 'Embrapa-48' na dose de 70 g/ha de diclosulam, a qual foi de $9,9 \mathrm{~cm}$ contra $12,1 \mathrm{~cm}$ na testemunha; e, na 'FT-Abyara' com flumetsulam a $240 \mathrm{~g} / \mathrm{ha}$ e em sua mistura com diclosulam. Em todos os tratamentos com herbicidas houve uma tendência discreta e geral de engrossamento do caule (Tabela 4), quando comparadas a testemunha sem produto, com destaque na 'FT-10 Princesa' em dose de $240 \mathrm{~g} / \mathrm{ha}$ de flumetsulam. O número de nódulos (Tabela 5) foi reduzido, principalmente nas doses dobradas de flumetsulam e diclosulam, nas cultivares 'FT-Guaira', 'Ocepar14 e 13', 'FT-10 Princesa' e 'Embrapa 4', exceto por diclosulam e por flumetsulam na 'RS-7 Jacuí'. $\mathrm{Na}$ 'Embrapa-4', 'RS-Jacuí', 'BR-36', 'FTGuaira' e 'Ocepar-14' o peso seco de nódulos (Tabela 6) também foi menor nas doses dobradas destes herbicidas. Nestes tratamentos, ainda, o peso seco por unidade de nódulo (Tabela 7) foi menor nas cultivares 'BR-16' e 'BR-36', sendo nesta última menor também com imazaquin, mais a 'FT-2000' com flumetsulam apenas. Ou seja, o tamanho dos nódulos foi menor. $\mathrm{O}$ destaque foi a cultivar 'Embrapa 4', que em todos os tratamentos com herbicida houve reduções e de forma decrescente com o aumento da dose, no número de nódulos. Nas demais cultivares a formação de nódulos foi indiferente ao uso de herbicidas, pois em certos casos houve até aumento significativo deles.

O peso da biomassa seca da parte aérea (Tabela 8) foi menor nas cultivares 'BR-36', 'Embrapa-48', com flumetsulam, e 'Ocepar 14' com diclosulam, principalmente em doses mais altas. As médias gerais de biomassa da parte superior das parcelas tratadas com herbicidas foram na maioria maiores ao da testemunha. 
TABELA 1. Número de plantas de soja por metro (estande), obtidas aos 17 dias após a emergência, de cultivares de soja de diferentes ciclos sob efeito de herbicidas, aplicados em pré-emergência. Londrina - PR, 1998.

\begin{tabular}{|c|c|c|c|c|c|c|c|c|c|c|c|c|c|c|}
\hline \multirow{2}{*}{\multicolumn{2}{|c|}{ TRATAMENTOS }} & \multicolumn{12}{|c|}{ CULTIVARES } & \multirow{3}{*}{$\begin{array}{c}\text { MÉDIA } \\
\text { GERAL } \\
\text { HERBICIDA }\end{array}$} \\
\hline & & \multicolumn{3}{|c|}{ PRECOCE } & \multicolumn{4}{|c|}{ SEMIPRECOCE } & \multicolumn{5}{|c|}{ MÉDIO } & \\
\hline HERBICIDA & $\begin{array}{c}\text { DOSE } \\
\text { g i.a/ha }\end{array}$ & $\begin{array}{c}\text { FT- } \\
\text { GUAIRA }\end{array}$ & $\begin{array}{c}\text { EMBRAPA- } \\
48\end{array}$ & $\begin{array}{c}\text { OCEPAR } \\
14\end{array}$ & BR-16 & $\begin{array}{c}\text { OCEPAR } \\
13\end{array}$ & $\begin{array}{c}\text { EMBRAPA- } \\
4\end{array}$ & BR-36 & $\begin{array}{c}\text { FT-10 } \\
\text { PRINCESA }\end{array}$ & $\begin{array}{c}\text { FT } \\
2000\end{array}$ & $\begin{array}{c}\text { FT } \\
\text { ABYARA }\end{array}$ & BR -37 & $\begin{array}{l}\text { RS-7 } \\
\text { JACUI }\end{array}$ & \\
\hline $\begin{array}{c}\text { Testemunha } \\
\text { capinada }\end{array}$ & -- & $\begin{array}{c}21,7 \mathrm{a} \\
\mathrm{A}\end{array}$ & $\begin{array}{c}18,5 \mathrm{a} \\
\mathrm{A}\end{array}$ & $\begin{array}{c}19,0 \mathrm{a} \\
\mathrm{A}\end{array}$ & $\begin{array}{c}21,0 \mathrm{a} \\
\mathrm{A}\end{array}$ & $\begin{array}{c}20,7 \mathrm{ab} \\
\mathrm{A}\end{array}$ & $\begin{array}{c}20,0 \mathrm{a} \\
\mathrm{A}\end{array}$ & $\begin{array}{c}22,7 \mathrm{a} \\
\mathrm{A}\end{array}$ & $\begin{array}{c}20,0 \mathrm{a} \\
\mathrm{A}\end{array}$ & $\begin{array}{c}18,7 \mathrm{a} \\
\mathrm{A}\end{array}$ & $\begin{array}{c}21,5 \mathrm{a} \\
\mathrm{A}\end{array}$ & $\begin{array}{c}22,0 \mathrm{a} \\
\mathrm{A}\end{array}$ & $\begin{array}{c}23,0 \mathrm{a} \\
\mathrm{A}\end{array}$ & $20,7 \mathrm{a}$ \\
\hline diclosulam & 35 & $\begin{array}{c}21,5 \mathrm{a} \\
\mathrm{A}\end{array}$ & $\begin{array}{c}20,7 \mathrm{a} \\
\mathrm{A}\end{array}$ & $\begin{array}{c}22,2 \mathrm{a} \\
\mathrm{A}\end{array}$ & $\begin{array}{c}21,2 \mathrm{a} \\
\mathrm{A}\end{array}$ & $\begin{array}{c}18,2 \mathrm{ab} \\
\mathrm{A}\end{array}$ & $\begin{array}{c}19,2 \mathrm{a} \\
\mathrm{A}\end{array}$ & $\begin{array}{c}20,5 \mathrm{a} \\
\mathrm{A}\end{array}$ & $\begin{array}{c}19,2 \text { a } \\
\text { A }\end{array}$ & $\begin{array}{c}18,7 \mathrm{a} \\
\mathrm{A}\end{array}$ & $\begin{array}{c}21,2 \mathrm{a} \\
\mathrm{A}\end{array}$ & $\begin{array}{c}20,5 \mathrm{a} \\
\mathrm{A}\end{array}$ & $\begin{array}{c}20,2 \mathrm{ab} \\
\mathrm{A}\end{array}$ & $20,3 \mathrm{a}$ \\
\hline diclosulam & 70 & $\begin{array}{l}21,2 \mathrm{a} \\
\mathrm{ABC}\end{array}$ & $\begin{array}{c}18,5 \mathrm{a} \\
\mathrm{BC}\end{array}$ & $\begin{array}{l}19,0 \mathrm{a} \\
\mathrm{ABC}\end{array}$ & $\begin{array}{c}23,5 \mathrm{a} \\
\mathrm{A}\end{array}$ & $\begin{array}{c}23,0 \mathrm{a} \\
\mathrm{AB}\end{array}$ & $\begin{array}{c}20,7 \mathrm{a} \\
\mathrm{ABC}\end{array}$ & $\begin{array}{c}21,7 \mathrm{a} \\
\mathrm{ABC}\end{array}$ & $\begin{array}{c}21,2 \mathrm{a} \\
\mathrm{ABC}\end{array}$ & $\begin{array}{c}17,7 \mathrm{a} \\
\mathrm{C}\end{array}$ & $\begin{array}{c}20,5 \mathrm{a} \\
\mathrm{ABC}\end{array}$ & $\begin{array}{l}19,5 \mathrm{a} \\
\mathrm{ABC}\end{array}$ & $\begin{array}{c}19,5 \mathrm{ab} \\
\mathrm{ABC}\end{array}$ & $20,5 \mathrm{a}$ \\
\hline flumetsulam & 120 & $\begin{array}{c}21,0 \mathrm{a} \\
\mathrm{A}\end{array}$ & $\begin{array}{c}19,2 \mathrm{a} \\
\mathrm{AB}\end{array}$ & $\begin{array}{c}18,0 \mathrm{a} \\
\mathrm{AB}\end{array}$ & $\begin{array}{c}18,5 \mathrm{a} \\
\mathrm{AB}\end{array}$ & $\begin{array}{c}22,7 \mathrm{a} \\
\mathrm{A}\end{array}$ & $\begin{array}{c}20,7 \mathrm{a} \\
\mathrm{A}\end{array}$ & $\begin{array}{c}21,2 \mathrm{a} \\
\mathrm{A}\end{array}$ & $\begin{array}{c}21,0 \mathrm{a} \\
\mathrm{A}\end{array}$ & $\begin{array}{c}18,2 \mathrm{a} \\
\mathrm{AB}\end{array}$ & $\begin{array}{c}18,5 \mathrm{a} \\
\mathrm{AB}\end{array}$ & $\begin{array}{c}22,5 \mathrm{a} \\
\mathrm{A}\end{array}$ & $\begin{array}{c}15,7 \mathrm{~b} \\
\mathrm{~B}\end{array}$ & $19,8 \mathrm{a}$ \\
\hline flumetsulam & 240 & $\begin{array}{c}19,7 \mathrm{a} \\
\mathrm{A}\end{array}$ & $\begin{array}{c}21,0 \mathrm{a} \\
\mathrm{A}\end{array}$ & $\begin{array}{c}20,2 \mathrm{a} \\
\mathrm{A}\end{array}$ & $\begin{array}{c}19,2 \mathrm{a} \\
\mathrm{A}\end{array}$ & $\begin{array}{c}17,7 \mathrm{ab} \\
\mathrm{A}\end{array}$ & $\begin{array}{c}18,0 \mathrm{a} \\
\mathrm{A}\end{array}$ & $\begin{array}{c}18,5 \mathrm{a} \\
\mathrm{A}\end{array}$ & $\begin{array}{c}20,5 \mathrm{a} \\
\mathrm{A}\end{array}$ & $\begin{array}{c}18,5 \mathrm{a} \\
\mathrm{A}\end{array}$ & $\begin{array}{c}17,2 \mathrm{a} \\
\mathrm{A}\end{array}$ & $\begin{array}{c}18,7 \mathrm{a} \\
\mathrm{A}\end{array}$ & $\begin{array}{c}21,2 \mathrm{ab} \\
\mathrm{A}\end{array}$ & $19,2 \mathrm{a}$ \\
\hline $\begin{array}{l}\text { diclosulam + } \\
\text { flumetsulam }\end{array}$ & $20+100$ & $\begin{array}{c}18,7 \mathrm{a} \\
\mathrm{AB}\end{array}$ & $\begin{array}{c}20,2 \mathrm{a} \\
\mathrm{AB}\end{array}$ & $\begin{array}{c}21,2 \mathrm{a} \\
\mathrm{A}\end{array}$ & $\begin{array}{c}20,0 \mathrm{a} \\
\mathrm{AB}\end{array}$ & $\begin{array}{c}16,2 \mathrm{~b} \\
\mathrm{~B}\end{array}$ & $\begin{array}{c}21,2 \mathrm{a} \\
\mathrm{A}\end{array}$ & $\begin{array}{c}20,5 \mathrm{a} \\
\mathrm{AB}\end{array}$ & $\begin{array}{c}19,7 \mathrm{a} \\
\mathrm{AB}\end{array}$ & $\begin{array}{c}19,0 \mathrm{a} \\
\mathrm{AB}\end{array}$ & $\begin{array}{c}18,7 \mathrm{a} \\
\mathrm{AB}\end{array}$ & $\begin{array}{c}19,7 \mathrm{a} \\
\mathrm{AB}\end{array}$ & $\begin{array}{c}20,7 \mathrm{ab} \\
\mathrm{AB}\end{array}$ & $19,7 \mathrm{a}$ \\
\hline imazaquin & 150 & $\begin{array}{c}22,5 \mathrm{a} \\
\mathrm{A}\end{array}$ & $\begin{array}{c}21,2 \mathrm{a} \\
\mathrm{AB}\end{array}$ & $\begin{array}{c}17,5 \mathrm{a} \\
\mathrm{B}\end{array}$ & $\begin{array}{c}19,2 \mathrm{a} \\
\mathrm{AB}\end{array}$ & $\begin{array}{c}20,0 \mathrm{ab} \\
\mathrm{AB}\end{array}$ & $\begin{array}{c}18,2 \mathrm{a} \\
\mathrm{AB}\end{array}$ & $\begin{array}{c}19,7 \mathrm{a} \\
\mathrm{AB}\end{array}$ & $\begin{array}{c}18,7 \mathrm{a} \\
\mathrm{AB}\end{array}$ & $\begin{array}{c}20,2 \mathrm{a} \\
\mathrm{AB}\end{array}$ & $\begin{array}{c}20,0 \mathrm{a} \\
\mathrm{AB}\end{array}$ & $\begin{array}{c}17,7 \mathrm{a} \\
\mathrm{AB}\end{array}$ & $\begin{array}{c}19,0 \mathrm{ab} \\
\mathrm{AB}\end{array}$ & $19,5 \mathrm{a}$ \\
\hline MÉDIA G & ERAL & 20,9 & 19,9 & 19,6 & 20,4 & 19,8 & 19,7 & 20,7 & 20,1 & 18,7 & 19,7 & 20,1 & 19,9 & -- \\
\hline CULTIV & ARES & A & $\mathrm{AB}$ & $\mathrm{AB}$ & $\mathrm{AB}$ & $\mathrm{AB}$ & $\mathrm{AB}$ & $\mathrm{AB}$ & $\mathrm{AB}$ & B & $\mathrm{AB}$ & $\mathrm{AB}$ & $\mathrm{AB}$ & \\
\hline
\end{tabular}

* Médias seguidas pela mesma letra não diferem entre si pelo Teste de Tukey ao nível de 5\% de probabilidade.

** Letras minúsculas a direita de cada valor, representam as médias dos tratamentos dentro de cada variedade e devem ser comparadas na vertical.

*** Letras maiúsculas sob cada valor, correspondem as médias de cultivares dentro de cada tratamento e devem ser comparadas na horizontal. 
TABELA 2. Altura (cm) de plantas de soja, quando $20 \%$ delas encontravam-se em florescimento (R2), de cultivares de soja de diferentes ciclos sob efeito de herbicidas, aplicados em pré-emergência. Londrina - PR, 1998.

\begin{tabular}{|c|c|c|c|c|c|c|c|c|c|c|c|c|c|c|}
\hline \multirow{2}{*}{\multicolumn{2}{|c|}{ TRATAMENTOS }} & \multicolumn{12}{|c|}{ CULTIVARES } & \multirow{3}{*}{$\begin{array}{c}\text { MÉDIA } \\
\text { GERAL } \\
\text { HERBICIDA }\end{array}$} \\
\hline & & \multicolumn{3}{|c|}{ PRECOCE } & \multicolumn{4}{|c|}{ SEMIPRECOCE } & \multicolumn{5}{|c|}{ MÉDIO } & \\
\hline HERBICIDA & $\begin{array}{c}\text { DOSE } \\
\text { g i.a/ha }\end{array}$ & $\begin{array}{c}\text { FT- } \\
\text { GUAIRA }\end{array}$ & $\begin{array}{c}\text { EMBRAPA- } \\
48\end{array}$ & $\begin{array}{c}\text { OCEPAR } \\
14\end{array}$ & BR-16 & $\begin{array}{c}\text { OCEPAR } \\
13\end{array}$ & $\begin{array}{c}\text { EMBRAPA- } \\
\quad 4\end{array}$ & BR-36 & $\begin{array}{c}\text { FT-10 } \\
\text { PRINCESA }\end{array}$ & $\begin{array}{c}\text { FT } \\
2000\end{array}$ & $\begin{array}{c}\text { FT } \\
\text { ABYARA }\end{array}$ & $\mathrm{BR}-37$ & $\begin{array}{l}\text { RS-7 } \\
\text { JACUI }\end{array}$ & \\
\hline $\begin{array}{c}\text { Testemunha } \\
\text { capinada }\end{array}$ & -- & $\begin{array}{c}85,07 \mathrm{a} \\
\mathrm{BC}\end{array}$ & $\begin{array}{c}88,37 \text { a } \\
\text { BC }\end{array}$ & $\begin{array}{c}87,67 \text { a } \\
\text { BC }\end{array}$ & $\begin{array}{c}91,32 \mathrm{a} \\
\text { B }\end{array}$ & $\begin{array}{c}76,85 \mathrm{a} \\
\mathrm{C}\end{array}$ & $\begin{array}{c}94,10 \mathrm{a} \\
\mathrm{B}\end{array}$ & $\begin{array}{c}81,82 \mathrm{a} \\
\text { BC }\end{array}$ & $\begin{array}{c}94,22 \mathrm{a} \\
\mathrm{B}\end{array}$ & $\begin{array}{c}124,47 \\
\mathrm{~b} \\
\mathrm{~A}\end{array}$ & $\begin{array}{c}75,60 \mathrm{a} \\
\mathrm{C}\end{array}$ & $\begin{array}{c}81,80 \text { a } \\
\text { BC }\end{array}$ & $\begin{array}{c}80,80 \mathrm{a} \\
\mathrm{BC}\end{array}$ & 88,51 a \\
\hline diclosulam & 35 & $\begin{array}{c}92,87 \mathrm{a} \\
\mathrm{BC}\end{array}$ & $\begin{array}{c}97,32 \mathrm{a} \\
\mathrm{B}\end{array}$ & $\begin{array}{l}84,07 \text { a } \\
\text { BCDE }\end{array}$ & $\begin{array}{c}91,70 \mathrm{a} \\
\text { BC }\end{array}$ & $\begin{array}{c}75,92 \mathrm{a} \\
\mathrm{DE}\end{array}$ & $\begin{array}{l}87,50 \text { a } \\
\text { BCDE }\end{array}$ & $\begin{array}{l}87,50 \mathrm{a} \\
\mathrm{BCDE}\end{array}$ & $\begin{array}{c}89,97 \text { a } \\
\text { BCD }\end{array}$ & $\begin{array}{c}122,87 \\
\mathrm{~b} \\
\mathrm{~A}\end{array}$ & $\begin{array}{c}74,47 \text { a } \\
\text { E }\end{array}$ & $\begin{array}{l}86,45 \text { a } \\
\text { BCDE }\end{array}$ & $\begin{array}{c}82,52 \mathrm{a} \\
\mathrm{CDE}\end{array}$ & 89,43 a \\
\hline diclosulam & 70 & $\begin{array}{c}91,45 \mathrm{a} \\
\text { BC }\end{array}$ & $\begin{array}{c}95,52 \mathrm{a} \\
\mathrm{B}\end{array}$ & $\begin{array}{c}78,00 \mathrm{a} \\
\mathrm{CD}\end{array}$ & $\begin{array}{c}92,12 \mathrm{a} \\
\mathrm{BC}\end{array}$ & $\begin{array}{c}80,62 \mathrm{a} \\
\mathrm{CD}\end{array}$ & $\begin{array}{c}87,75 \text { a } \\
\text { BCD }\end{array}$ & $\begin{array}{c}78,77 \text { a } \\
\mathrm{CD}\end{array}$ & $\begin{array}{c}95,92 \mathrm{a} \\
\text { B }\end{array}$ & $\begin{array}{c}132,85 \\
\mathrm{~b} \\
\mathrm{~A}\end{array}$ & $\begin{array}{c}77,17 \text { a } \\
\text { D }\end{array}$ & $\begin{array}{c}88,12 \text { a } \\
\text { BCD }\end{array}$ & $\begin{array}{c}81,97 \text { a } \\
\text { BCD }\end{array}$ & 90,02 a \\
\hline flumetsulam & 120 & $\begin{array}{c}95,30 \mathrm{a} \\
\mathrm{BC}\end{array}$ & $\begin{array}{c}97,82 \text { a } \\
\text { B }\end{array}$ & $\begin{array}{l}87,15 \mathrm{a} \\
\mathrm{BCDE}\end{array}$ & $\begin{array}{c}91,35 \mathrm{a} \\
\mathrm{BCD}\end{array}$ & $\begin{array}{c}80,77 \text { a } \\
\text { DE }\end{array}$ & $\begin{array}{c}89,30 \mathrm{a} \\
\mathrm{BCD}\end{array}$ & $\begin{array}{c}80,07 \mathrm{a} \\
\mathrm{DE}\end{array}$ & $\begin{array}{c}89,05 \text { a } \\
\text { BCD }\end{array}$ & $\begin{array}{c}123,67 \\
\mathrm{~b} \\
\mathrm{~A}\end{array}$ & $\begin{array}{c}73,60 \mathrm{a} \\
\mathrm{E}\end{array}$ & $\begin{array}{c}82,62 \text { a } \\
\mathrm{CDE}\end{array}$ & $\begin{array}{c}78,42 \mathrm{a} \\
\mathrm{DE}\end{array}$ & 89,09 a \\
\hline flumetsulam & 240 & $\begin{array}{c}87,75 \text { a } \\
\text { BCD }\end{array}$ & $\begin{array}{c}89,15 \text { a } \\
\text { BC }\end{array}$ & $\begin{array}{c}87,20 \text { a } \\
\text { BCD }\end{array}$ & $\begin{array}{c}93,75 \mathrm{a} \\
\text { B }\end{array}$ & $\begin{array}{c}71,55 \mathrm{a} \\
\mathrm{E}\end{array}$ & $\begin{array}{c}91,35 \mathrm{a} \\
\mathrm{BC}\end{array}$ & $\begin{array}{c}78,00 \mathrm{a} \\
\mathrm{CDE}\end{array}$ & $\begin{array}{c}88,55 \text { a } \\
\text { BC }\end{array}$ & $\begin{array}{c}131,20 \\
\mathrm{~b} \\
\mathrm{~A}\end{array}$ & $\begin{array}{c}73,85 \text { a } \\
\text { DE }\end{array}$ & $\begin{array}{l}82,20 \text { a } \\
\text { BCDE }\end{array}$ & $\begin{array}{l}80,85 \text { a } \\
\text { BCDE }\end{array}$ & 87,95 a \\
\hline $\begin{array}{l}\text { diclosulam + } \\
\text { flumetsulam }\end{array}$ & $20+100$ & $\begin{array}{c}95,57 \mathrm{a} \\
\mathrm{B}\end{array}$ & $\begin{array}{c}93,05 \mathrm{a} \\
\mathrm{B}\end{array}$ & $\begin{array}{c}84,55 \mathrm{a} \\
\mathrm{BCD}\end{array}$ & $\begin{array}{c}90,42 \mathrm{a} \\
\text { BC }\end{array}$ & $\begin{array}{c}76,62 \mathrm{a} \\
\mathrm{CD}\end{array}$ & $\begin{array}{c}88,50 \mathrm{a} \\
\text { BC }\end{array}$ & $\begin{array}{c}84,57 \mathrm{a} \\
\mathrm{BCD}\end{array}$ & $\begin{array}{c}94,77 \text { a } \\
\text { B }\end{array}$ & $\begin{array}{c}151,62 \text { a } \\
\text { A }\end{array}$ & $\begin{array}{c}71,12 \mathrm{a} \\
\mathrm{D}\end{array}$ & $\begin{array}{c}82,47 \text { a } \\
\text { BCD }\end{array}$ & $\begin{array}{c}82,02 \mathrm{a} \\
\mathrm{BCD}\end{array}$ & $91,28 \mathrm{a}$ \\
\hline imazaquin & 150 & $\begin{array}{c}84,57 \text { a } \\
\text { BCD }\end{array}$ & $\begin{array}{c}93,32 \text { a } \\
\text { B }\end{array}$ & $\begin{array}{c}88,40 \text { a } \\
\text { BCD }\end{array}$ & $\begin{array}{c}90,04 \mathrm{a} \\
\mathrm{BC}\end{array}$ & $\begin{array}{c}79,15 \text { a } \\
\mathrm{CD}\end{array}$ & $\begin{array}{c}85,05 \text { a } \\
\text { BCD }\end{array}$ & $\begin{array}{c}76,65 \text { a } \\
C D\end{array}$ & $\begin{array}{c}93,67 \text { a } \\
\text { B }\end{array}$ & $\begin{array}{c}132,67 \\
\mathrm{~b} \\
\mathrm{~A}\end{array}$ & $\begin{array}{c}75,75 \mathrm{a} \\
\mathrm{D}\end{array}$ & $\begin{array}{c}83,72 \text { a } \\
\text { BCD }\end{array}$ & $\begin{array}{c}75,72 \mathrm{a} \\
\mathrm{D}\end{array}$ & 88,23 a \\
\hline MÉDIA G & ERAL & 90,37 & 93,51 & 85,29 & 91,53 & 77,36 & 89,08 & 81,06 & 92,31 & 131,34 & 74,51 & 83,91 & 80,33 & -- \\
\hline CULTIV & ARES & $\mathrm{BCD}$ & $\mathrm{B}$ & $\mathrm{CDE}$ & $\mathrm{BC}$ & FG & $\mathrm{BCD}$ & $\mathrm{EF}$ & B & A & G & $\mathrm{DE}$ & EFG & \\
\hline
\end{tabular}

* Médias seguidas pela mesma letra não diferem entre si pelo Teste de Tukey ao nível de 5\% de probabilidade.

** Letras minúsculas a direita de cada valor, representam as médias dos tratamentos dentro de cada variedade e devem ser comparadas na vertical.

*** Letras maiúsculas sob cada valor, correspondem as médias de cultivares dentro de cada tratamento e devem ser comparadas na horizontal. 
TABELA 3. Inserção (cm) da primeira vagem, realizada por ocasião da colheita, de cultivares de soja de diferentes ciclos sob efeito de herbicidas, aplicados em pré-emergência. Londrina - PR, 1998.

\begin{tabular}{|c|c|c|c|c|c|c|c|c|c|c|c|c|c|c|}
\hline \multirow{2}{*}{\multicolumn{2}{|c|}{ TRATAMENTOS }} & \multicolumn{12}{|c|}{ CULTIVARES } & \multirow{3}{*}{$\begin{array}{c}\text { MÉDIA } \\
\text { GERAL } \\
\text { HERBICIDA }\end{array}$} \\
\hline & & \multicolumn{3}{|c|}{ PRECOCE } & \multicolumn{4}{|c|}{ SEMIPRECOCE } & \multicolumn{5}{|c|}{ MÉDIO } & \\
\hline HERBICIDA & $\begin{array}{c}\text { DOSE } \\
\text { g i.a/ha }\end{array}$ & $\begin{array}{c}\text { FT- } \\
\text { GUAIRA }\end{array}$ & $\begin{array}{c}\text { EMBRAPA- } \\
48\end{array}$ & $\begin{array}{c}\text { OCEPAR } \\
14\end{array}$ & BR-16 & $\begin{array}{c}\text { OCEPAR } \\
13\end{array}$ & $\begin{array}{c}\text { EMBRAPA- } \\
4\end{array}$ & BR-36 & $\begin{array}{c}\text { FT-10 } \\
\text { PRINCESA }\end{array}$ & $\begin{array}{c}\text { FT } \\
2000\end{array}$ & $\begin{array}{c}\text { FT } \\
\text { ABYARA }\end{array}$ & BR-37 & $\begin{array}{l}\text { RS-7 } \\
\text { JACUI }\end{array}$ & \\
\hline $\begin{array}{c}\text { Testemunha } \\
\text { capinada }\end{array}$ & -- & $\begin{array}{c}13,9 \mathrm{a} \\
\mathrm{ABC}\end{array}$ & $\begin{array}{l}12,1 \mathrm{a} \\
\mathrm{BCD}\end{array}$ & $\begin{array}{c}10,1 \mathrm{a} \\
\mathrm{D}\end{array}$ & $\begin{array}{c}16,0 \text { a } \\
\text { A }\end{array}$ & $\begin{array}{c}14,6 \mathrm{a} \\
\mathrm{AB}\end{array}$ & $\begin{array}{l}13,1 \mathrm{a} \\
\mathrm{ABCD}\end{array}$ & $\begin{array}{l}12,0 \mathrm{a} \\
\mathrm{BCD}\end{array}$ & $\begin{array}{c}11,1 \mathrm{a} \\
\mathrm{CD}\end{array}$ & $\begin{array}{l}11,4 \mathrm{a} \\
\mathrm{BCD}\end{array}$ & $\begin{array}{c}16,4 \mathrm{a} \\
\mathrm{A}\end{array}$ & $\begin{array}{l}11,3 \mathrm{a} \\
\mathrm{BCD}\end{array}$ & $\begin{array}{l}13,4 \mathrm{a} \\
\mathrm{ABCD}\end{array}$ & $13,0 \mathrm{a}$ \\
\hline diclosulam & 35 & $\begin{array}{c}11,9 \mathrm{a} \\
\mathrm{BC}\end{array}$ & $\begin{array}{c}10,5 \mathrm{a} \\
\mathrm{C}\end{array}$ & $\begin{array}{c}11,8 \mathrm{a} \\
\mathrm{BC}\end{array}$ & $\begin{array}{c}16,4 \mathrm{a} \\
\mathrm{A}\end{array}$ & $\begin{array}{c}13,5 \mathrm{a} \\
\mathrm{ABC}\end{array}$ & $\begin{array}{c}11,7 \mathrm{a} \\
\mathrm{BC}\end{array}$ & $\begin{array}{c}13,0 \mathrm{a} \\
\mathrm{ABC}\end{array}$ & $\begin{array}{c}12,3 \mathrm{a} \\
\mathrm{BC}\end{array}$ & $\begin{array}{c}10,9 \mathrm{a} \\
\mathrm{C}\end{array}$ & $\begin{array}{c}14,4 \mathrm{a} \\
\mathrm{AB}\end{array}$ & $\begin{array}{c}11,9 \mathrm{a} \\
\mathrm{BC}\end{array}$ & $\begin{array}{c}12,7 \mathrm{a} \\
\mathrm{BC}\end{array}$ & $12,6 \mathrm{a}$ \\
\hline diclosulam & 70 & $\begin{array}{c}15,0 \mathrm{a} \\
\mathrm{AB}\end{array}$ & $\begin{array}{c}9,9 \mathrm{a} \\
\mathrm{C} \\
\end{array}$ & $\begin{array}{c}10,4 \mathrm{a} \\
\mathrm{C} \\
\end{array}$ & $\begin{array}{c}15,4 \text { a } \\
\mathrm{A} \\
\end{array}$ & $\begin{array}{c}14,2 \mathrm{a} \\
\mathrm{AB} \\
\end{array}$ & $\begin{array}{c}11,7 \mathrm{a} \\
\mathrm{BC}\end{array}$ & $\begin{array}{l}12,7 \mathrm{a} \\
\mathrm{ABC}\end{array}$ & $\begin{array}{l}12,9 \mathrm{a} \\
\mathrm{ABC} \\
\end{array}$ & $\begin{array}{c}13,3 \mathrm{a} \\
\mathrm{ABC}\end{array}$ & $\begin{array}{c}15,9 \mathrm{a} \\
\mathrm{A} \\
\end{array}$ & $\begin{array}{c}11,6 \mathrm{a} \\
\mathrm{BC} \\
\end{array}$ & $\begin{array}{c}13,3 \mathrm{a} \\
\mathrm{ABC}\end{array}$ & $13,0 \mathrm{a}$ \\
\hline flumetsulam & 120 & $\begin{array}{c}12,8 \mathrm{a} \\
\mathrm{BC}\end{array}$ & $\begin{array}{c}10,2 \mathrm{a} \\
\mathrm{C}\end{array}$ & $\begin{array}{c}10,4 \mathrm{a} \\
\mathrm{C}\end{array}$ & $\begin{array}{c}17,4 \text { a } \\
\text { A }\end{array}$ & $\begin{array}{c}16,3 \mathrm{a} \\
\mathrm{A}\end{array}$ & $\begin{array}{c}12,3 \mathrm{a} \\
\mathrm{BC}\end{array}$ & $\begin{array}{c}12,5 \mathrm{a} \\
\mathrm{BC}\end{array}$ & $\begin{array}{c}12,3 \mathrm{a} \\
\mathrm{BC}\end{array}$ & $\begin{array}{c}14,2 \mathrm{a} \\
\mathrm{BC}\end{array}$ & $\begin{array}{c}14,2 \mathrm{a} \\
\mathrm{AB}\end{array}$ & $\begin{array}{c}11,8 \mathrm{a} \\
\mathrm{BC}\end{array}$ & $\begin{array}{c}12,5 \mathrm{a} \\
\mathrm{BC}\end{array}$ & $13,1 \mathrm{a}$ \\
\hline flumetsulam & 240 & $\begin{array}{l}14,0 \mathrm{a} \\
\mathrm{ABC}\end{array}$ & $\begin{array}{l}12,6 \mathrm{a} \\
\mathrm{ABC}\end{array}$ & $\begin{array}{c}10,7 \mathrm{a} \\
\mathrm{BC}\end{array}$ & $\begin{array}{c}15,2 \mathrm{a} \\
\mathrm{A}\end{array}$ & $\begin{array}{l}13,6 \text { a } \\
\mathrm{ABC}\end{array}$ & $\begin{array}{c}14,2 \mathrm{a} \\
\mathrm{AB}\end{array}$ & $\begin{array}{l}11,8 \mathrm{a} \\
\mathrm{ABC}\end{array}$ & $\begin{array}{c}10,5 \mathrm{a} \\
\mathrm{C}\end{array}$ & $\begin{array}{l}13,9 \mathrm{a} \\
\mathrm{ABC}\end{array}$ & $\begin{array}{c}13,3 \mathrm{a} \\
\mathrm{ABC}\end{array}$ & $\begin{array}{l}12,8 \mathrm{a} \\
\mathrm{ABC}\end{array}$ & $\begin{array}{l}12,6 \mathrm{a} \\
\mathrm{ABC}\end{array}$ & $12,9 \mathrm{a}$ \\
\hline $\begin{array}{l}\text { diclosulam + } \\
\text { flumetsulam }\end{array}$ & $20+100$ & $\begin{array}{c}13,3 \mathrm{a} \\
\mathrm{AB}\end{array}$ & $\begin{array}{c}12,5 \mathrm{a} \\
\mathrm{AB}\end{array}$ & $\begin{array}{c}11,9 \mathrm{a} \\
\mathrm{B}\end{array}$ & $\begin{array}{c}15,7 \mathrm{a} \\
\mathrm{A}\end{array}$ & $\begin{array}{c}14,2 \mathrm{a} \\
\mathrm{AB}\end{array}$ & $\begin{array}{c}12,7 \mathrm{a} \\
\mathrm{AB}\end{array}$ & $\begin{array}{c}12,4 \mathrm{a} \\
\mathrm{AB}\end{array}$ & $\begin{array}{c}11,2 \mathrm{a} \\
\mathrm{B}\end{array}$ & $\begin{array}{c}13,3 \mathrm{a} \\
\mathrm{AB}\end{array}$ & $\begin{array}{c}13,3 \mathrm{a} \\
\mathrm{AB}\end{array}$ & $\begin{array}{c}11,6 \mathrm{a} \\
\mathrm{B}\end{array}$ & $\begin{array}{c}12,5 \mathrm{a} \\
\mathrm{AB}\end{array}$ & $12,9 \mathrm{a}$ \\
\hline imazaquin & 150 & $\begin{array}{l}12,3 \mathrm{a} \\
\mathrm{ABC}\end{array}$ & $\begin{array}{c}11,2 \mathrm{a} \\
\mathrm{BC}\end{array}$ & $\begin{array}{l}12,8 \mathrm{a} \\
\mathrm{ABC}\end{array}$ & $\begin{array}{l}14,3 \mathrm{a} \\
\mathrm{ABC}\end{array}$ & $\begin{array}{c}14,4 \mathrm{a} \\
\mathrm{AB}\end{array}$ & $\begin{array}{c}11,6 \mathrm{a} \\
\mathrm{BC}\end{array}$ & $\begin{array}{l}12,4 \mathrm{a} \\
\mathrm{ABC}\end{array}$ & $\begin{array}{l}12,2 \mathrm{a} \\
\mathrm{ABC}\end{array}$ & $\begin{array}{l}14,1 \mathrm{a} \\
\mathrm{ABC}\end{array}$ & $\begin{array}{c}15,6 \mathrm{a} \\
\mathrm{A} \\
\end{array}$ & $\begin{array}{c}10,9 \mathrm{a} \\
\mathrm{C}\end{array}$ & $\begin{array}{l}12,4 \mathrm{a} \\
\mathrm{ABC}\end{array}$ & $12,9 \mathrm{a}$ \\
\hline MÉDIA G & ERAL & 13,3 & 11,3 & 11,2 & 15,8 & 14,4 & 12,5 & 12,4 & 11,8 & 13,0 & 14,8 & 11,7 & 12,8 & -- \\
\hline CULTIV & ARES & $\mathrm{BCD}$ & $\mathrm{D}$ & $\mathrm{D}$ & A & $\mathrm{ABC}$ & $\mathrm{CD}$ & $\mathrm{CD}$ & $\mathrm{D}$ & $\mathrm{BCD}$ & $\mathrm{AB}$ & $\mathrm{D}$ & $\mathrm{BCD}$ & \\
\hline
\end{tabular}

* Médias seguidas pela mesma letra não diferem entre si pelo Teste de Tukey ao nível de 5\% de probabilidade.

** Letras minúsculas a direita de cada valor, representam as médias dos tratamentos dentro de cada variedade e devem ser comparadas na vertical.

*** Letras maiúsculas sob cada valor, correspondem as médias de cultivares dentro de cada tratamento e devem ser comparadas na horizontal. 
TABELA 4. Diâmetros ( $\mathrm{mm}$ ) dos caules de soja, obtidos por ocasião da colheita, de cultivares de soja de diferentes ciclos sob efeito de herbicidas, aplicados em pré-emergência. Londrina - PR, 1998.

\begin{tabular}{|c|c|c|c|c|c|c|c|c|c|c|c|c|c|c|}
\hline \multirow{2}{*}{\multicolumn{2}{|c|}{ TRATAMENTOS }} & \multicolumn{12}{|c|}{ CULTIVARES } & \multirow{3}{*}{$\begin{array}{c}\text { MÉDIA } \\
\text { GERAL } \\
\text { HERBICIDA }\end{array}$} \\
\hline & & \multicolumn{3}{|c|}{ PRECOCE } & \multicolumn{4}{|c|}{ SEMIPRECOCE } & \multicolumn{5}{|c|}{ MÉDIO } & \\
\hline HERBICIDA & $\begin{array}{c}\text { DOSE } \\
\text { g i.a/ha }\end{array}$ & $\begin{array}{c}\text { FT- } \\
\text { GUAIRA }\end{array}$ & $\begin{array}{l}\text { EMBRAPA- } \\
48 \\
\end{array}$ & $\begin{array}{c}\text { OCEPAR } \\
14 \\
\end{array}$ & BR-16 & $\begin{array}{c}\text { OCEPAR } \\
13 \\
\end{array}$ & $\begin{array}{c}\text { EMBRAPA- } \\
4 \\
\end{array}$ & BR-36 & $\begin{array}{c}\text { FT-10 } \\
\text { PRINCESA }\end{array}$ & $\begin{array}{c}\text { FT } \\
2000 \\
\end{array}$ & $\begin{array}{c}\text { FT } \\
\text { ABYARA }\end{array}$ & BR-37 & $\begin{array}{l}\text { RS-7 } \\
\text { JACUI }\end{array}$ & \\
\hline $\begin{array}{c}\text { Testemunha } \\
\text { capinada }\end{array}$ & -- & 6,212 & 6,662 & 6,337 & 6,602 & 6,525 & 6,655 & 6,524 & 8,341 & 7,880 & 5,915 & 6,262 & 6,515 & 6,703 \\
\hline diclosulam & 35 & 6,862 & 6,705 & 6,175 & 7,087 & 6,476 & 7,077 & 6,672 & 8,704 & 7,749 & 6,225 & 6,560 & 6,462 & 6,896 \\
\hline diclosulam & 70 & 6,350 & 6,790 & 6,662 & 7,287 & 6,865 & 6,727 & 6,456 & 8,955 & 7,994 & 6,157 & 7,162 & 6,552 & 6,997 \\
\hline flumetsulam & 120 & 6,225 & 6,626 & 6,212 & 6,699 & 6,585 & 6,660 & 6,505 & 8,344 & 8,045 & 6,146 & 6,410 & 6,642 & 6,758 \\
\hline flumetsulam & 240 & 6,587 & 6,460 & 6,312 & 7,179 & 6,434 & 6,687 & 6,509 & 10,330 & 7,807 & 6,125 & 6,361 & 6,860 & 6,971 \\
\hline $\begin{array}{l}\text { diclosulam + } \\
\text { flumetsulam }\end{array}$ & $20+100$ & 6,662 & 6,465 & 6,300 & 6,497 & 6,482 & 6,666 & 6,442 & 8,967 & 7,976 & 6,074 & 6,179 & 6,852 & 6,797 \\
\hline imazaquin & 150 & 6,712 & 6,701 & 6,225 & 6,699 & 6,394 & 6,720 & 6,631 & 9,085 & 7,957 & 6,256 & 6,626 & 6,705 & 6,893 \\
\hline $\begin{array}{l}\text { MÉDIA G } \\
\text { CULTIV }\end{array}$ & $\begin{array}{l}\text { ERAL } \\
\text { ARES }\end{array}$ & 6,516 & 6,630 & 6,318 & 6,864 & 6,537 & 6,742 & 6,534 & 8,961 & 7,915 & 6,128 & 6,509 & 6,656 & -- \\
\hline
\end{tabular}

* Dados não submetidos à análise de variância por não adequar a seus pressupostos. 
TABELA 5. Número de nódulos de 10 plantas de soja, quando $20 \%$ delas encontravam-se em florescimento (R2), de cultivares de soja de diferentes ciclos sob efeito de herbicidas, aplicados em pré-emergência. Londrina - PR, 1998.

\begin{tabular}{|c|c|c|c|c|c|c|c|c|c|c|c|c|c|c|}
\hline \multirow{2}{*}{\multicolumn{2}{|c|}{ TRATAMENTOS }} & \multicolumn{12}{|c|}{ CULTIVARES } & \multirow{3}{*}{$\begin{array}{c}\text { MÉDIA } \\
\text { GERAL } \\
\text { HERBICIDA }\end{array}$} \\
\hline & & \multicolumn{3}{|c|}{ PRECOCE } & \multicolumn{4}{|c|}{ SEMIPRECOCE } & \multicolumn{5}{|c|}{ MÉDIO } & \\
\hline HERBICIDA & $\begin{array}{c}\text { DOSE } \\
\text { g i.a/ha } \\
\end{array}$ & $\begin{array}{c}\text { FT- } \\
\text { GUAIRA }\end{array}$ & $\begin{array}{c}\text { EMBRAPA- } \\
48\end{array}$ & $\begin{array}{c}\text { OCEPAR } \\
14 \\
\end{array}$ & BR-16 & $\begin{array}{c}\text { OCEPAR } \\
13 \\
\end{array}$ & $\begin{array}{c}\text { EMBRAPA- } \\
4\end{array}$ & BR-36 & $\begin{array}{c}\text { FT-10 } \\
\text { PRINCESA }\end{array}$ & $\begin{array}{c}\text { FT } \\
2000 \\
\end{array}$ & $\begin{array}{c}\text { FT } \\
\text { ABYARA } \\
\end{array}$ & BR-37 & $\begin{array}{c}\text { RS-7 } \\
\text { JACUI } \\
\end{array}$ & \\
\hline $\begin{array}{c}\text { Testemunha } \\
\text { capinada }\end{array}$ & -- & 299 & 345 & 320 & 563 & 401 & 314 & 196 & 444 & 418 & 335 & 244 & 352 & 353 \\
\hline diclosulam & 35 & 231 & 660 & 254 & 526 & 372 & 309 & 223 & 389 & 534 & 346 & 256 & 267 & 364 \\
\hline diclosulam & 70 & 249 & 588 & 219 & 665 & 351 & 305 & 206 & 398 & 731 & 372 & 326 & 265 & 390 \\
\hline flumetsulam & 120 & 316 & 458 & 306 & 556 & 362 & 277 & 172 & 435 & 587 & 295 & 248 & 359 & 364 \\
\hline flumetsulam & 240 & 240 & 445 & 215 & 700 & 228 & 230 & 184 & 341 & 665 & 340 & 391 & 319 & 358 \\
\hline $\begin{array}{l}\text { diclosulam + } \\
\text { flumetsulam }\end{array}$ & $20+100$ & 284 & 611 & 287 & 601 & 374 & 248 & 222 & 352 & 470 & 263 & 379 & 394 & 374 \\
\hline imazaquin & 150 & 253 & 643 & 360 & 667 & 342 & 265 & 248 & 539 & 586 & 515 & 280 & 486 & 432 \\
\hline $\begin{array}{l}\text { MÉDIA G } \\
\text { CULTIV }\end{array}$ & $\begin{array}{l}\text { ERAL } \\
\text { ARES }\end{array}$ & 267 & 536 & 280 & 611 & 347 & 278 & 207 & 414 & 570 & 352 & 303 & 349 & -- \\
\hline
\end{tabular}

* Dados não submetidos à análise de variância por não adequar a seus pressupostos. 
TABELA 6. Peso (g) da biomassa seca de nódulos de 10 plantas de soja, por ocasião do florescimento (R2), de cultivares de soja de diferentes ciclos sob efeito de herbicidas, aplicados em pré-emergência. Londrina - PR, 1998.

\begin{tabular}{|c|c|c|c|c|c|c|c|c|c|c|c|c|c|c|}
\hline \multirow{2}{*}{\multicolumn{2}{|c|}{ TRATAMENTOS }} & \multicolumn{12}{|c|}{ CULTIVARES } & \multirow{3}{*}{$\begin{array}{c}\text { MÉDIA } \\
\text { GERAL } \\
\text { HERBICIDA }\end{array}$} \\
\hline & & \multicolumn{3}{|c|}{ PRECOCE } & \multicolumn{4}{|c|}{ SEMIPRECOCE } & \multicolumn{5}{|c|}{ MÉDIO } & \\
\hline HERBICIDA & $\begin{array}{c}\text { DOSE } \\
\mathrm{g} \mathrm{i.a/ha}\end{array}$ & $\begin{array}{c}\text { FT- } \\
\text { GUAIRA }\end{array}$ & $\begin{array}{c}\text { EMBRAPA- } \\
48\end{array}$ & $\begin{array}{c}\text { OCEPAR } \\
14\end{array}$ & BR-16 & $\begin{array}{c}\text { OCEPAR } \\
13\end{array}$ & $\begin{array}{c}\text { EMBRAPA- } \\
\quad 4\end{array}$ & BR-36 & $\begin{array}{c}\text { FT-10 } \\
\text { PRINCESA }\end{array}$ & $\begin{array}{c}\text { FT } \\
2000\end{array}$ & $\begin{array}{c}\text { FT } \\
\text { ABYARA }\end{array}$ & BR-37 & $\begin{array}{c}\text { RS-7 } \\
\text { JACUI }\end{array}$ & \\
\hline $\begin{array}{c}\text { Testemunha } \\
\text { capinada }\end{array}$ & -- & $\begin{array}{c}0,96 \mathrm{a} \\
\mathrm{BC}\end{array}$ & $\begin{array}{c}0,74 \mathrm{a} \\
\mathrm{C}\end{array}$ & $\begin{array}{c}0,73 \mathrm{a} \\
\mathrm{C}\end{array}$ & $\begin{array}{c}2,49 \mathrm{~b} \\
\mathrm{~A}\end{array}$ & $\begin{array}{c}1,19 \mathrm{a} \\
\mathrm{BC}\end{array}$ & $\begin{array}{c}0,91 \mathrm{a} \\
\mathrm{BC}\end{array}$ & $\begin{array}{c}0,93 \mathrm{a} \\
\mathrm{BC}\end{array}$ & $\begin{array}{c}1,44 \mathrm{a} \\
\mathrm{BC}\end{array}$ & $\begin{array}{c}1,69 \mathrm{~b} \\
\mathrm{AB}\end{array}$ & $\begin{array}{c}0,73 \mathrm{a} \\
\mathrm{C}\end{array}$ & $\begin{array}{c}0,80 \mathrm{a} \\
\mathrm{C}\end{array}$ & $\begin{array}{c}1,21 \mathrm{a} \\
\mathrm{BC}\end{array}$ & $1,15 \mathrm{ab}$ \\
\hline diclosulam & 35 & $\begin{array}{c}0,64 \mathrm{a} \\
\mathrm{C}\end{array}$ & $\begin{array}{c}1,41 \mathrm{a} \\
\mathrm{BC}\end{array}$ & $\begin{array}{c}0,73 \mathrm{a} \\
\mathrm{C}\end{array}$ & $\begin{array}{c}2,44 \mathrm{~b} \\
\mathrm{~A}\end{array}$ & $\begin{array}{c}1,17 \mathrm{a} \\
\mathrm{BC}\end{array}$ & $\begin{array}{c}0,97 \mathrm{a} \\
\mathrm{C}\end{array}$ & $\begin{array}{c}0,89 \mathrm{a} \\
\mathrm{C}\end{array}$ & $\begin{array}{c}1,27 \mathrm{a} \\
\mathrm{BC}\end{array}$ & $\begin{array}{c}1,87 \mathrm{ab} \\
\mathrm{AB}\end{array}$ & $\begin{array}{c}0,78 \mathrm{a} \\
\mathrm{C}\end{array}$ & $\begin{array}{c}0,81 \mathrm{a} \\
\mathrm{C}\end{array}$ & $\begin{array}{c}1,08 \mathrm{a} \\
\mathrm{BC}\end{array}$ & $1,18 \mathrm{ab}$ \\
\hline diclosulam & 70 & $\begin{array}{c}0,73 \mathrm{a} \\
\mathrm{B}\end{array}$ & $\begin{array}{c}1,32 \mathrm{a} \\
\mathrm{B}\end{array}$ & $\begin{array}{c}0,66 \mathrm{a} \\
\mathrm{B}\end{array}$ & $\begin{array}{c}2,55 \mathrm{ab} \\
\mathrm{A}\end{array}$ & $\begin{array}{c}1,09 \mathrm{a} \\
\mathrm{B}\end{array}$ & $\begin{array}{c}0,91 \mathrm{a} \\
\mathrm{B}\end{array}$ & $\begin{array}{c}0,64 \mathrm{a} \\
\mathrm{B}\end{array}$ & $\begin{array}{c}1,13 \mathrm{a} \\
\mathrm{B}\end{array}$ & $\begin{array}{c}2,60 \mathrm{a} \\
\mathrm{A}\end{array}$ & $\begin{array}{c}0,92 \mathrm{a} \\
\mathrm{B}\end{array}$ & $\begin{array}{c}1,18 \mathrm{a} \\
\mathrm{B}\end{array}$ & $\begin{array}{c}0,98 \mathrm{a} \\
\mathrm{B}\end{array}$ & $1,23 a b$ \\
\hline flumetsulam & 120 & $\begin{array}{c}1,04 \mathrm{a} \\
\mathrm{CD}\end{array}$ & $\begin{array}{c}0,95 \text { a } \\
\text { CD }\end{array}$ & $\begin{array}{c}0,99 \text { a } \\
\text { CD }\end{array}$ & $\begin{array}{c}2,40 \mathrm{~b} \\
\mathrm{~A}\end{array}$ & $\begin{array}{c}1,28 \mathrm{a} \\
\mathrm{CD}\end{array}$ & $\begin{array}{c}0,69 \mathrm{a} \\
\mathrm{D}\end{array}$ & $\begin{array}{c}0,66 \mathrm{a} \\
\mathrm{D}\end{array}$ & $\begin{array}{c}1,55 \mathrm{a} \\
\mathrm{BC}\end{array}$ & $\begin{array}{c}2,10 \mathrm{ab} \\
\mathrm{AB}\end{array}$ & $\begin{array}{c}0,80 \mathrm{a} \\
\mathrm{CD}\end{array}$ & $\begin{array}{c}0,77 \mathrm{a} \\
\mathrm{CD} \\
\end{array}$ & $\begin{array}{c}1,09 \mathrm{a} \\
\mathrm{CD}\end{array}$ & $1,19 \mathrm{ab}$ \\
\hline flumetsulam & 240 & $\begin{array}{c}0,87 \mathrm{a} \\
\mathrm{B} \\
\end{array}$ & $\begin{array}{c}0,77 \text { a } \\
\text { B } \\
\end{array}$ & $\begin{array}{c}0,74 \mathrm{a} \\
\mathrm{B}\end{array}$ & $\begin{array}{c}2,36 \mathrm{~b} \\
\mathrm{~A}\end{array}$ & $\begin{array}{c}0,73 \mathrm{a} \\
\mathrm{B}\end{array}$ & $\begin{array}{c}0,66 \mathrm{a} \\
\mathrm{B}\end{array}$ & $\begin{array}{c}0,66 \mathrm{a} \\
\mathrm{B}\end{array}$ & $\begin{array}{c}1,20 \mathrm{a} \\
\mathrm{B}\end{array}$ & $\begin{array}{c}2,14 \mathrm{ab} \\
\mathrm{A}\end{array}$ & $\begin{array}{c}0,79 \mathrm{a} \\
\mathrm{B}\end{array}$ & $\begin{array}{c}1,24 \mathrm{a} \\
\mathrm{B}\end{array}$ & $\begin{array}{c}1,12 \mathrm{a} \\
\mathrm{B}\end{array}$ & $1,11 \mathrm{~b}$ \\
\hline $\begin{array}{l}\text { diclosulam + } \\
\text { flumetsulam }\end{array}$ & $\begin{array}{c}20+10 \\
0\end{array}$ & $\begin{array}{c}0,98 \mathrm{a} \\
\mathrm{BC}\end{array}$ & $\begin{array}{c}1,07 \mathrm{a} \\
\mathrm{BC}\end{array}$ & $\begin{array}{c}0,71 \mathrm{a} \\
\mathrm{C}\end{array}$ & $\begin{array}{c}2,29 \mathrm{~b} \\
\mathrm{~A}\end{array}$ & $\begin{array}{c}1,21 \mathrm{a} \\
\mathrm{BC}\end{array}$ & $\begin{array}{c}0,54 \mathrm{a} \\
\mathrm{C}\end{array}$ & $\begin{array}{c}0,85 \mathrm{a} \\
\mathrm{BC}\end{array}$ & $\begin{array}{c}1,20 \mathrm{a} \\
\mathrm{BC}\end{array}$ & $\begin{array}{c}1,55 \mathrm{~b} \\
\mathrm{AB}\end{array}$ & $\begin{array}{c}0,64 \mathrm{a} \\
\mathrm{C}\end{array}$ & $\begin{array}{c}1,22 \mathrm{a} \\
\mathrm{BC}\end{array}$ & $\begin{array}{c}1,25 \mathrm{a} \\
\mathrm{BC}\end{array}$ & $1,13 \mathrm{ab}$ \\
\hline imazaquin & 150 & $\begin{array}{c}0,95 \mathrm{a} \\
\mathrm{D}\end{array}$ & $\begin{array}{c}1,22 \mathrm{a} \\
\mathrm{CD}\end{array}$ & $\begin{array}{c}0,79 \mathrm{a} \\
\mathrm{D}\end{array}$ & $\begin{array}{c}3,38 \mathrm{a} \\
\mathrm{A}\end{array}$ & $\begin{array}{c}1,06 \mathrm{a} \\
\mathrm{CD}\end{array}$ & $\begin{array}{c}0,92 \text { a } \\
\text { D }\end{array}$ & $\begin{array}{c}0,85 \mathrm{a} \\
\mathrm{D}\end{array}$ & $\begin{array}{c}1,78 \mathrm{a} \\
\mathrm{BC}\end{array}$ & $\begin{array}{c}2,19 \mathrm{ab} \\
\mathrm{B} \\
\end{array}$ & $\begin{array}{c}1,23 \mathrm{a} \\
\mathrm{CD}\end{array}$ & $\begin{array}{c}0,85 \mathrm{a} \\
\mathrm{D}\end{array}$ & $\begin{array}{l}1,54 \mathrm{a} \\
\mathrm{BCD}\end{array}$ & $1,40 \mathrm{a}$ \\
\hline $\begin{array}{l}\text { MÉDIA G } \\
\text { CULTIV }\end{array}$ & $\begin{array}{l}\text { ERAL } \\
\text { ARES }\end{array}$ & $\begin{array}{r}0,90 \\
\mathrm{CDE}\end{array}$ & $\begin{array}{c}1,07 \\
\text { BCDE }\end{array}$ & $\begin{array}{c}0,76 \\
\mathrm{E}\end{array}$ & $\begin{array}{c}2,56 \\
\mathrm{~A}\end{array}$ & $\begin{array}{r}1,11 \\
\text { BCD }\end{array}$ & $\begin{array}{c}0,80 \\
\mathrm{DE}\end{array}$ & $\begin{array}{c}0,78 \\
\mathrm{E}\end{array}$ & $\begin{array}{c}1,37 \\
\text { B }\end{array}$ & $\begin{array}{c}2,02 \\
\text { A }\end{array}$ & $\begin{array}{r}0,84 \\
\mathrm{DE}\end{array}$ & $\begin{array}{c}0,98 \\
\text { BCDE }\end{array}$ & $\begin{array}{l}1,18 \\
\mathrm{BC}\end{array}$ & -- \\
\hline
\end{tabular}

* Médias seguidas pela mesma letra não diferem entre si pelo Teste de Tukey ao nível de 5\% de probabilidade.

** Letras minúsculas a direita de cada valor, representam as médias dos tratamentos dentro de cada variedade e devem ser comparadas na vertical.

*** Letras maiúsculas sob cada valor, correspondem as médias de cultivares dentro de cada tratamento e devem ser comparadas na horizontal.

**** Dados originais foram transformados em raiz quadrada de $\mathrm{y}$. 
TABELA 7. Peso (mg) da biomassa seca por unidade de nódulos, por ocasião do florescimento (R2), de cultivares de soja de diferentes ciclos sob efeito de herbicidas, aplicados em pré-emergência. Londrina - PR, 1998.

\begin{tabular}{|c|c|c|c|c|c|c|c|c|c|c|c|c|c|c|}
\hline \multirow{2}{*}{\multicolumn{2}{|c|}{ TRATAMENTOS }} & \multicolumn{12}{|c|}{ CULTIVARES } & \multirow{3}{*}{$\begin{array}{c}\text { MÉDIA } \\
\text { GERAL } \\
\text { HERBICIDA }\end{array}$} \\
\hline & & \multicolumn{3}{|c|}{ PRECOCE } & \multicolumn{4}{|c|}{ SEMIPRECOCE } & \multicolumn{5}{|c|}{ MÉDIO } & \\
\hline HERBICIDA & $\begin{array}{c}\text { DOSE } \\
\text { g i.a/ha }\end{array}$ & $\begin{array}{c}\text { FT- } \\
\text { GUAIRA }\end{array}$ & $\begin{array}{c}\text { EMBRAPA- } \\
48\end{array}$ & $\begin{array}{c}\text { OCEPAR } \\
14\end{array}$ & BR-16 & $\begin{array}{c}\text { OCEPAR } \\
13\end{array}$ & $\begin{array}{c}\text { EMBRAPA- } \\
4\end{array}$ & BR-36 & $\begin{array}{c}\text { FT-10 } \\
\text { PRINCESA }\end{array}$ & $\begin{array}{c}\text { FT } \\
2000\end{array}$ & $\begin{array}{c}\text { FT } \\
\text { ABYARA }\end{array}$ & BR-37 & $\begin{array}{c}\text { RS-7 } \\
\text { JACUI }\end{array}$ & \\
\hline $\begin{array}{c}\text { Testemunha } \\
\text { capinada }\end{array}$ & -- & $\begin{array}{c}3,26 \mathrm{a} \\
\mathrm{ABC}\end{array}$ & $\begin{array}{c}2,09 \mathrm{a} \\
\mathrm{C}\end{array}$ & $\begin{array}{c}2,33 \mathrm{a} \\
\mathrm{C}\end{array}$ & $\begin{array}{c}4,32 \mathrm{ab} \\
\mathrm{AB}\end{array}$ & $\begin{array}{c}2,90 \mathrm{a} \\
\mathrm{BC}\end{array}$ & $\begin{array}{c}2,98 \mathrm{a} \\
\mathrm{ABC}\end{array}$ & $\begin{array}{c}4,60 \mathrm{a} \\
\mathrm{A}\end{array}$ & $\begin{array}{c}3,31 \mathrm{a} \\
\mathrm{ABC}\end{array}$ & $\begin{array}{c}4,03 \mathrm{a} \\
\mathrm{AB}\end{array}$ & $\begin{array}{c}2,33 \mathrm{a} \\
\mathrm{C}\end{array}$ & $\begin{array}{c}3,21 \mathrm{a} \\
\mathrm{ABC}\end{array}$ & $\begin{array}{c}3,54 \mathrm{a} \\
\mathrm{ABC}\end{array}$ & $3,24 \mathrm{a}$ \\
\hline diclosulam & 35 & $\begin{array}{c}3,28 \mathrm{a} \\
\mathrm{ABC}\end{array}$ & $\begin{array}{c}2,10 \mathrm{a} \\
\mathrm{C}\end{array}$ & $\begin{array}{c}3,05 \mathrm{a} \\
\mathrm{BC}\end{array}$ & $\begin{array}{c}4,85 \mathrm{ab} \\
\mathrm{A}\end{array}$ & $\begin{array}{l}3,20 \mathrm{a} \\
\mathrm{ABC}\end{array}$ & $\begin{array}{l}3,13 \mathrm{a} \\
\mathrm{ABC}\end{array}$ & $\begin{array}{c}4,08 \mathrm{a} \\
\mathrm{AB}\end{array}$ & $\begin{array}{c}3,21 \mathrm{a} \\
\mathrm{ABC}\end{array}$ & $\begin{array}{c}3,56 \mathrm{a} \\
\mathrm{ABC}\end{array}$ & $\begin{array}{c}2,26 \mathrm{a} \\
\mathrm{C}\end{array}$ & $\begin{array}{c}3,11 \mathrm{a} \\
\mathrm{BC}\end{array}$ & $\begin{array}{c}4,05 \mathrm{a} \\
\mathrm{AB}\end{array}$ & $3,32 \mathrm{a}$ \\
\hline diclosulam & 70 & $\begin{array}{c}3,07 \mathrm{a} \\
\mathrm{AB}\end{array}$ & $\begin{array}{c}2,20 \mathrm{a} \\
\mathrm{B}\end{array}$ & $\begin{array}{c}2,90 \mathrm{a} \\
\mathrm{AB}\end{array}$ & $\begin{array}{c}3,91 \mathrm{ab} \\
\mathrm{A}\end{array}$ & $\begin{array}{c}3,27 \mathrm{a} \\
\mathrm{AB}\end{array}$ & $\begin{array}{c}3,15 \mathrm{a} \\
\mathrm{AB}\end{array}$ & $\begin{array}{c}3,55 \mathrm{a} \\
\mathrm{AB}\end{array}$ & $\begin{array}{c}3,34 \mathrm{a} \\
\mathrm{AB}\end{array}$ & $\begin{array}{c}3,71 \mathrm{a} \\
\mathrm{AB}\end{array}$ & $\begin{array}{c}2,54 \mathrm{a} \\
\mathrm{AB}\end{array}$ & $\begin{array}{c}3,63 \mathrm{a} \\
\mathrm{AB}\end{array}$ & $\begin{array}{c}3,72 \mathrm{a} \\
\mathrm{AB}\end{array}$ & $3,25 \mathrm{a}$ \\
\hline flumetsulam & 120 & $\begin{array}{c}3,17 \mathrm{a} \\
\mathrm{AB}\end{array}$ & $\begin{array}{c}2,12 \mathrm{a} \\
\mathrm{B}\end{array}$ & $\begin{array}{c}3,46 \mathrm{a} \\
\mathrm{AB}\end{array}$ & $\begin{array}{c}4,16 \mathrm{ab} \\
\mathrm{A}\end{array}$ & $\begin{array}{c}3,52 \mathrm{a} \\
\mathrm{AB}\end{array}$ & $\begin{array}{c}3,24 \mathrm{a} \\
\mathrm{AB}\end{array}$ & $\begin{array}{c}3,94 \mathrm{a} \\
\mathrm{A}\end{array}$ & $\begin{array}{c}3,99 \text { a } \\
\text { A }\end{array}$ & $\begin{array}{c}3,62 \mathrm{a} \\
\mathrm{AB}\end{array}$ & $\begin{array}{c}2,58 \mathrm{a} \\
\mathrm{AB}\end{array}$ & $\begin{array}{c}3,07 \mathrm{a} \\
\mathrm{AB}\end{array}$ & $\begin{array}{c}3,10 \mathrm{a} \\
\mathrm{AB}\end{array}$ & $3,33 \mathrm{a}$ \\
\hline flumetsulam & 240 & $\begin{array}{c}3,67 \mathrm{a} \\
\mathrm{A}\end{array}$ & $\begin{array}{c}1,83 \mathrm{a} \\
\mathrm{B}\end{array}$ & $\begin{array}{c}3,34 \mathrm{a} \\
\mathrm{AB}\end{array}$ & $\begin{array}{c}3,42 \mathrm{~b} \\
\mathrm{AB}\end{array}$ & $\begin{array}{c}3,45 \mathrm{a} \\
\mathrm{AB}\end{array}$ & $\begin{array}{c}3,03 \mathrm{a} \\
\mathrm{AB}\end{array}$ & $\begin{array}{c}3,44 \mathrm{a} \\
\mathrm{AB}\end{array}$ & $\begin{array}{c}3,37 \mathrm{a} \\
\mathrm{AB}\end{array}$ & $\begin{array}{c}3,22 \mathrm{a} \\
\mathrm{AB}\end{array}$ & $\begin{array}{c}2,32 \mathrm{a} \\
\mathrm{AB}\end{array}$ & $\begin{array}{c}3,20 \mathrm{a} \\
\mathrm{AB}\end{array}$ & $\begin{array}{c}3,63 \mathrm{a} \\
\mathrm{A}\end{array}$ & $3,16 \mathrm{a}$ \\
\hline $\begin{array}{l}\text { diclosulam + } \\
\text { flumetsulam }\end{array}$ & $\begin{array}{c}20+10 \\
0\end{array}$ & $\begin{array}{c}3,60 \mathrm{a} \\
\mathrm{A}\end{array}$ & $\begin{array}{c}1,76 \mathrm{a} \\
\mathrm{B}\end{array}$ & $\begin{array}{c}2,51 \mathrm{a} \\
\mathrm{AB}\end{array}$ & $\begin{array}{c}3,92 \mathrm{ab} \\
\mathrm{A}\end{array}$ & $\begin{array}{c}3,38 \mathrm{a} \\
\mathrm{AB}\end{array}$ & $\begin{array}{c}2,74 \mathrm{a} \\
\mathrm{AB}\end{array}$ & $\begin{array}{c}3,94 \mathrm{a} \\
\mathrm{A}\end{array}$ & $\begin{array}{c}3,48 \text { a } \\
\text { A }\end{array}$ & $\begin{array}{c}3,42 \mathrm{a} \\
\mathrm{AB}\end{array}$ & $\begin{array}{c}2,40 \mathrm{a} \\
\mathrm{AB}\end{array}$ & $\begin{array}{c}3,17 \mathrm{a} \\
\mathrm{AB}\end{array}$ & $\begin{array}{c}3,21 \mathrm{a} \\
\mathrm{AB}\end{array}$ & $3,13 \mathrm{a}$ \\
\hline imazaquin & 150 & $\begin{array}{c}3,67 \mathrm{a} \\
\mathrm{AB}\end{array}$ & $\begin{array}{c}2,24 \mathrm{a} \\
\mathrm{B}\end{array}$ & $\begin{array}{c}2,30 \mathrm{a} \\
\mathrm{B}\end{array}$ & $\begin{array}{c}4,99 \mathrm{a} \\
\mathrm{A}\end{array}$ & $\begin{array}{c}3,05 \text { a } \\
\text { B }\end{array}$ & $\begin{array}{c}3,89 \mathrm{a} \\
\mathrm{AB}\end{array}$ & $\begin{array}{c}3,24 \mathrm{a} \\
\mathrm{B}\end{array}$ & $\begin{array}{c}3,23 \mathrm{a} \\
\mathrm{Bb}\end{array}$ & $\begin{array}{c}3,75 \mathrm{a} \\
\mathrm{AB}\end{array}$ & $\begin{array}{c}2,40 \mathrm{a} \\
\mathrm{B}\end{array}$ & $\begin{array}{c}3,09 \mathrm{a} \\
\mathrm{B}\end{array}$ & $\begin{array}{c}3,11 \mathrm{a} \\
\mathrm{B}\end{array}$ & $3,25 \mathrm{a}$ \\
\hline MÉDIA G & ERAL & 3,39 & 2,05 & 2,84 & 4,22 & 3,25 & 3,17 & 3,83 & 3,42 & 3,61 & 2,40 & 3,21 & 3,48 & -- \\
\hline CULTIV & ARES & $\mathrm{BCD}$ & $\mathrm{F}$ & $\mathrm{DE}$ & $\mathrm{A}$ & $\mathrm{BCD}$ & $\mathrm{CD}$ & $\mathrm{AB}$ & $\mathrm{BCD}$ & $\mathrm{ABC}$ & $\mathrm{EF}$ & $\mathrm{BCD}$ & $\mathrm{BC}$ & \\
\hline
\end{tabular}

* Médias seguidas pela mesma letra não diferem entre si pelo Teste de Tukey ao nível de 5\% de probabilidade.

** Letras minúsculas a direita de cada valor, representam as médias dos tratamentos dentro de cada variedade e devem ser comparadas na vertical.

*** Letras maiúsculas sob cada valor, correspondem as médias de cultivares dentro de cada tratamento e devem ser comparadas na horizontal.

***** Dados originais foram transformados em $\mathrm{y}^{0.573}$. 
A biomassa do sistema radicular (Tabela 9) de nenhuma das cultivares foi reduzida. $\mathrm{Na}$ realidade houve até aumento de peso na maioria.

Nenhuma das cultivares exibiu significante suscetibilidade aos tratamentos herbicidas, em todas as variáveis individuais estudadas, sendo isto evidente nas doses normais (menores doses) dos dois herbicidas utilizados. Porém, em doses dobradas, em algumas cultivares, pode-se notar certos efeitos negativos, mas também sem significância. Nas observações visuais não foram notados sintomas de fitotoxicidade às plantas de soja em nenhum dos tratamentos. Os rendimentos (Tabela 10), variável considerada de importância máxima, das cultivares 'FT-Guaira', 'Embrapa-4' e 'BR-37' foram reduzidos em 20,9\%, 11,8\% e 11,0\% respectivamente, em relação à testemunha, quando se utilizou $70 \mathrm{~g} / \mathrm{ha}$ de diclosulam. Nesta dose, a FT-Guaira teve ainda o número (Tabela 5) e peso de nódulos (Tabela 6) diminuídos, talvez sendo a causa de seu menor rendimento; a Embrapa-4 teve a altura de plantas e inserção da primeira vagem reduzidos; enquanto a BR-37 foi afetada no estande. $\mathrm{O}$ rendimento da cultivar 'BR-37' também foi ligeiramente reduzido pelo diclosulam à $35 \mathrm{~g} / \mathrm{ha}$, dando indicações de a mesma apresentar certa sensibilidade ao produto. Os rendimentos destas três cultivares, e também da 'Ocepar-14', foram afetados em percentuais menores também pelo flumetsulam na dose de $240 \mathrm{~g} / \mathrm{ha}$ e pela mistura do flumetsulam + diclosulam $(100+20 \mathrm{~g} / \mathrm{ha})$. Ao passo que as cultivares 'FT-Guaíra' e Embrapa-4, com as doses normais de diclosulam ( $35 \mathrm{~g} / \mathrm{ha}$ ) e de flumetsulam (120 g/ha) sozinhos, assim como as demais cultivares, em doses normais e dobradas, não tiveram seus rendimentos afetados, assemelhandose à testemunha mantida no limpo pela capina. Em relação ao imazaquin na dose de $150 \mathrm{~g} / \mathrm{ha}$, apenas a cultivar 'FT-Guaíra' teve o rendimento reduzido em $6 \%$, enquanto o das demais cultivares foram superiores ao da testemunha.

Apesar de todas estas informações obtidas neste experimento, não foi possível distinguir nenhuma cultivar suscetível ou com pelo menos em alguma de suas características agronômicas alteradas pelos herbicidas flumetsulam e diclosulam. Em relação ao primeiro herbicida, os resultados estão de acordo com Bronhara \& Mengarda (1993) e Rocha (1993); que baseados em experimento de campo afirmam ser o flumetsulam seletivo para a cultura da soja, quando utilizado em doses normais recomendadas. Mas Magalhães \& Barros (1993) e Guimarães \& Valente (1995), também em experimento de campo, registraram injúrias temporárias na soja, a qual se apresentou com clorose foliar e redução de crescimento. Quanto ao diclosulam Kruse et al. (1997), Pelissari et al. (1997) e Zagonel et al. (1997) relatam não terem observados sinais de injúria nas cultivares de soja 'BR-16', 'FTGuaira', e 'Ocepar-14' respectivamente quando utilizaram doses de até $40 \mathrm{~g} / \mathrm{ha}$.

Esta relativa tolerância demonstrada por todas as cultivares, independente da base genética, ou do grupo de maturação a que pertencem, indica serem possuidoras de características semelhantes no que se refere a seletividade as estes herbicidas, podendo isto estar associado ao que relatam Hodges et al. (1990) de que a seletividade do flumetsulam é em função do tempo requerido para absorção e translocação do mesmo, e do metabolismo diferencial. Portanto, se o herbicida for absorvido e translocado lentamente e em pequenas doses, há chances para um melhor metabolismo, e consequentemente mais baixa é a possibilidade de fitotoxicidade às plantas de soja. Porém, se determinadas condições, como pH elevado e ou baixo teor de matéria orgânica, favorecerem pronta disponibilidade do herbicida na solução do solo, altas taxas de absorção podem ocorrer mais rapidamente e, então, provocar fitotoxicação à soja, uma vez que o mecanismo de metabolização é sobrecarregado. Os mesmos autores relatam também que a seletividade de um importante representante das sulfonanilidas (herbicida "A") é devida a detoxificação metabólica, em razão de hidroxilação (reação de oxidação) seguida de conjugação com glicose. 
TABELA 8. Peso (g) da biomassa seca da parte aérea de 10 plantas de soja, por ocasião do florescimento (R2), de cultivares de soja de diferentes ciclos sob efeito de herbicidas, aplicados em pré-emergência. Londrina - PR, 1998.

\begin{tabular}{|c|c|c|c|c|c|c|c|c|c|c|c|c|c|c|}
\hline \multirow{2}{*}{\multicolumn{2}{|c|}{ TRATAMENTOS }} & \multicolumn{12}{|c|}{ CULTIVARES } & \multirow{3}{*}{$\begin{array}{c}\text { MÉDIA } \\
\text { GERAL } \\
\text { HERBICIDA }\end{array}$} \\
\hline & & \multicolumn{3}{|c|}{ PRECOCE } & \multicolumn{4}{|c|}{ SEMIPRECOCE } & \multicolumn{5}{|c|}{ MÉDIO } & \\
\hline HERBICIDA & $\begin{array}{c}\text { DOSE } \\
\mathrm{g} \mathrm{i.a/ha}\end{array}$ & $\begin{array}{c}\text { FT- } \\
\text { GUAIRA }\end{array}$ & $\begin{array}{c}\text { EMBRAPA- } \\
48 \\
\end{array}$ & $\begin{array}{c}\text { OCEPAR } \\
14 \\
\end{array}$ & BR-16 & $\begin{array}{c}\text { OCEPAR } \\
13 \\
\end{array}$ & $\begin{array}{l}\text { EMBRAPA- } \\
\quad 4\end{array}$ & BR-36 & $\begin{array}{c}\text { FT-10 } \\
\text { PRINCESA }\end{array}$ & $\begin{array}{c}\text { FT } \\
2000\end{array}$ & $\begin{array}{c}\text { FT } \\
\text { ABYARA }\end{array}$ & BR-37 & $\begin{array}{c}\text { RS-7 } \\
\text { JACUI }\end{array}$ & \\
\hline $\begin{array}{l}\text { Testemunha } \\
\text { capinada }\end{array}$ & -- & $\begin{array}{c}88,74 \text { a } \\
\text { BC }\end{array}$ & $\begin{array}{c}117,68 \mathrm{ab} \\
\mathrm{ABC}\end{array}$ & $\begin{array}{c}82,38 \text { a } \\
\quad C\end{array}$ & $\begin{array}{c}126,22 \mathrm{ab} \\
\mathrm{AB}\end{array}$ & $\begin{array}{c}105,77 \mathrm{a} \\
\mathrm{BC}\end{array}$ & $\begin{array}{c}100,76 \mathrm{~b} \\
\mathrm{BC}\end{array}$ & $\begin{array}{c}89,56 \text { a } \\
\text { BC }\end{array}$ & $\begin{array}{c}117,39 \mathrm{a} \\
\mathrm{ABC}\end{array}$ & $\begin{array}{c}149,62 \mathrm{a} \\
\mathrm{A}\end{array}$ & $\begin{array}{c}100,98 \text { a } \\
\text { BC }\end{array}$ & $\begin{array}{c}96,31 \text { a } \\
\text { BC }\end{array}$ & $\begin{array}{c}97,89 \text { a } \\
\text { BC }\end{array}$ & $106,11 \mathrm{a}$ \\
\hline diclosulam & 35 & $\begin{array}{c}109,69 \text { a } \\
A B\end{array}$ & $\begin{array}{c}110,32 \mathrm{ab} \\
\mathrm{AB}\end{array}$ & $\begin{array}{c}78,25 \mathrm{a} \\
\mathrm{B}\end{array}$ & $\begin{array}{c}116,61 \mathrm{~b} \\
\mathrm{AB}\end{array}$ & $\begin{array}{c}130,55 \mathrm{a} \\
\mathrm{A}\end{array}$ & $\begin{array}{c}133,01 \mathrm{ab} \\
\mathrm{A}\end{array}$ & $\begin{array}{c}97,74 \mathrm{a} \\
\mathrm{AB}\end{array}$ & $\begin{array}{c}129,35 \mathrm{a} \\
\mathrm{A}\end{array}$ & $\begin{array}{c}123,17 \mathrm{a} \\
\mathrm{A}\end{array}$ & $\begin{array}{c}93,44 \text { a } \\
\text { A }\end{array}$ & $\begin{array}{c}94,56 \mathrm{a} \\
\mathrm{AB}\end{array}$ & $\begin{array}{c}94,51 \mathrm{a} \\
\mathrm{AB}\end{array}$ & $109,27 \mathrm{a}$ \\
\hline diclosulam & 70 & $\begin{array}{c}95,77 \mathrm{a} \\
\mathrm{BC}\end{array}$ & $\begin{array}{c}108,53 \mathrm{ab} \\
\mathrm{BC}\end{array}$ & $\begin{array}{c}75,03 \mathrm{a} \\
\mathrm{C}\end{array}$ & $\begin{array}{c}121,85 \mathrm{ab} \\
\mathrm{AB}\end{array}$ & $\begin{array}{c}120,30 \mathrm{a} \\
\mathrm{AB}\end{array}$ & $\begin{array}{c}111,77 \mathrm{ab} \\
\mathrm{BC}\end{array}$ & $\begin{array}{c}105,30 \mathrm{a} \\
\mathrm{BC}\end{array}$ & $\begin{array}{c}113,59 \mathrm{a} \\
\mathrm{BC}\end{array}$ & $\begin{array}{c}155,37 \text { a } \\
\text { A }\end{array}$ & $\begin{array}{c}96,10 \mathrm{a} \\
\mathrm{BC}\end{array}$ & $\begin{array}{c}113,39 \mathrm{a} \\
\text { BC }\end{array}$ & $\begin{array}{c}98,14 \mathrm{a} \\
\mathrm{BC}\end{array}$ & $109,60 \mathrm{a}$ \\
\hline flumetsulam & 120 & $\begin{array}{c}97,36 \mathrm{a} \\
\mathrm{BC}\end{array}$ & $\begin{array}{c}111,22 \mathrm{ab} \\
\mathrm{BC}\end{array}$ & $\begin{array}{c}99,72 \text { a } \\
\text { BC }\end{array}$ & $\begin{array}{c}159,53 \text { a } \\
\text { A }\end{array}$ & $\begin{array}{c}110,61 \mathrm{a} \\
\text { BC }\end{array}$ & $\begin{array}{c}106,84 \mathrm{~b} \\
\mathrm{BC}\end{array}$ & $\begin{array}{c}73,62 \mathrm{a} \\
\mathrm{C}\end{array}$ & $\begin{array}{c}119,38 \text { a } \\
\text { AB }\end{array}$ & $\begin{array}{c}154,14 \text { a } \\
\text { A }\end{array}$ & $\begin{array}{c}103,25 \mathrm{a} \\
\text { BC }\end{array}$ & $\begin{array}{c}108,33 \text { a } \\
\text { BC }\end{array}$ & $\begin{array}{c}121,69 \mathrm{a} \\
\mathrm{AB}\end{array}$ & $103,81 \mathrm{a}$ \\
\hline flumetsulam & 240 & $\begin{array}{c}89,78 \text { a } \\
\text { C }\end{array}$ & $\begin{array}{c}95,88 \mathrm{~b} \\
\mathrm{C}\end{array}$ & $\begin{array}{c}85,69 \text { a } \\
\text { C }\end{array}$ & $\begin{array}{c}150,36 \mathrm{ab} \\
\mathrm{A}\end{array}$ & $\begin{array}{c}117,63 \mathrm{a} \\
\mathrm{ABC}\end{array}$ & $\begin{array}{c}105,58 \mathrm{~b} \\
\mathrm{BC}\end{array}$ & $\begin{array}{c}80,02 \mathrm{a} \\
\mathrm{C}\end{array}$ & $\begin{array}{c}140,43 \mathrm{a} \\
\mathrm{AB}\end{array}$ & $\begin{array}{c}155,76 \mathrm{a} \\
\mathrm{A}\end{array}$ & $\begin{array}{c}105,13 \mathrm{a} \\
\mathrm{BC}\end{array}$ & $\begin{array}{c}100,86 \text { a } \\
\text { BC }\end{array}$ & $\begin{array}{c}103,41 \mathrm{a} \\
\mathrm{BC}\end{array}$ & $110,88 \mathrm{a}$ \\
\hline $\begin{array}{l}\text { diclosulam + } \\
\text { flumetsulam }\end{array}$ & $20+100$ & $\begin{array}{c}94,54 \mathrm{a} \\
\mathrm{BC}\end{array}$ & $\begin{array}{c}107,19 \mathrm{ab} \\
\mathrm{ABC}\end{array}$ & $\begin{array}{c}87,56 \mathrm{a} \\
\mathrm{C}\end{array}$ & $\begin{array}{c}133,35 \mathrm{ab} \\
\mathrm{AB}\end{array}$ & $\begin{array}{c}124,23 \mathrm{a} \\
\mathrm{ABC}\end{array}$ & $\begin{array}{c}106,69 b \\
\mathrm{ABC}\end{array}$ & $\begin{array}{c}95,13 \mathrm{a} \\
\mathrm{BC}\end{array}$ & $\begin{array}{c}114,65 \mathrm{a} \\
\mathrm{ABC}\end{array}$ & $\begin{array}{c}147,22 \text { a } \\
\text { A }\end{array}$ & $\begin{array}{c}100,52 \mathrm{a} \\
\mathrm{BC}\end{array}$ & $\begin{array}{c}106,76 \text { a } \\
\text { ABC }\end{array}$ & $\begin{array}{c}122,70 \mathrm{a} \\
\mathrm{ABC}\end{array}$ & $111,71 \mathrm{a}$ \\
\hline imazaquin & 150 & $\begin{array}{c}72,43 \text { a } \\
\text { D }\end{array}$ & $\begin{array}{c}144,91 \mathrm{a} \\
\mathrm{AB}\end{array}$ & $\begin{array}{c}85,79 \text { a } \\
\text { CD }\end{array}$ & $\begin{array}{c}127,21 \mathrm{ab} \\
\mathrm{ABC}\end{array}$ & $\begin{array}{c}113,35 \mathrm{a} \\
\mathrm{ABCD}\end{array}$ & $\begin{array}{c}153,86 \text { a } \\
\text { A }\end{array}$ & $\begin{array}{c}90,73 \mathrm{a} \\
\mathrm{CD}\end{array}$ & $\begin{array}{c}117,09 \mathrm{a} \\
\mathrm{ABC}\end{array}$ & $\begin{array}{c}153,07 \mathrm{a} \\
\mathrm{A}\end{array}$ & $\begin{array}{c}104,20 \mathrm{a} \\
\text { BCD }\end{array}$ & $\begin{array}{c}114,32 \text { a } \\
\text { ABC }\end{array}$ & $\begin{array}{c}119,04 \mathrm{a} \\
\mathrm{ABC}\end{array}$ & $116,33 \mathrm{a}$ \\
\hline MÉDIA G & RAL & 92,62 & 113,68 & 84,92 & 133,59 & 117,49 & 116,93 & 90,30 & 121,70 & 148,34 & 100,52 & 104,93 & 108,20 & -- \\
\hline CULTIVA & RES & EFG & $\mathrm{CD}$ & $\mathrm{E}$ & $\mathrm{AB}$ & $\mathrm{BCD}$ & $\mathrm{BCD}$ & FG & $\mathrm{BC}$ & A & DEF & $\mathrm{CDE}$ & $\mathrm{CDE}$ & \\
\hline
\end{tabular}

* Médias seguidas pela mesma letra não diferem entre si pelo Teste de Tukey ao nível de 5\% de probabilidade.

** Letras minúsculas a direita de cada valor, representam as médias dos tratamentos dentro de cada variedade e devem ser comparadas na vertical.

*** Letras maiúsculas sob cada valor, correspondem as médias de cultivares dentro de cada tratamento e devem ser comparadas na horizontal.

**** Dados originais foram transformados em $\log (\mathrm{y})$. 
TABELA 9. Peso (g) da biomassa seca de raízes de 10 plantas de soja, por ocasião do florescimento (R2), de cultivares de soja de diferentes ciclos sob efeito de herbicidas, aplicados em pré-emergência. Londrina - PR, 1998.

\begin{tabular}{|c|c|c|c|c|c|c|c|c|c|c|c|c|c|c|}
\hline \multirow{2}{*}{\multicolumn{2}{|c|}{ TRATAMENTOS }} & \multicolumn{12}{|c|}{ CULTIVARES } & \multirow{3}{*}{$\begin{array}{c}\text { MÉDIA } \\
\text { GERAL } \\
\text { HERBICIDA }\end{array}$} \\
\hline & & \multicolumn{3}{|c|}{ PRECOCE } & \multicolumn{4}{|c|}{ SEMIPRECOCE } & \multicolumn{5}{|c|}{ MÉDIO } & \\
\hline HERBICIDA & $\begin{array}{c}\text { DOSE } \\
\text { g i.a/ha }\end{array}$ & $\begin{array}{c}\text { FT- } \\
\text { GUAIRA }\end{array}$ & $\begin{array}{c}\text { EMBRAPA- } \\
48 \\
\end{array}$ & $\begin{array}{c}\text { OCEPAR } \\
14 \\
\end{array}$ & BR-16 & $\begin{array}{c}\text { OCEPAR } \\
13 \\
\end{array}$ & $\begin{array}{l}\text { EMBRAPA- } \\
\quad 4 \\
\end{array}$ & BR-36 & $\begin{array}{c}\text { FT-10 } \\
\text { PRINCESA } \\
\end{array}$ & $\begin{array}{c}\text { FT } \\
2000 \\
\end{array}$ & $\begin{array}{c}\text { FT } \\
\text { ABYARA }\end{array}$ & BR-37 & $\begin{array}{c}\text { RS-7 } \\
\text { JACUI } \\
\end{array}$ & \\
\hline $\begin{array}{c}\text { Testemunha } \\
\text { capinada }\end{array}$ & -- & $\begin{array}{c}7,96 \mathrm{a} \\
\mathrm{C}\end{array}$ & $\begin{array}{c}9,71 \mathrm{a} \\
\mathrm{BC}\end{array}$ & $\begin{array}{c}7,43 \mathrm{a} \\
\mathrm{C}\end{array}$ & $\begin{array}{c}12,14 \mathrm{a} \\
\mathrm{AB}\end{array}$ & $\begin{array}{c}9,52 \mathrm{a} \\
\mathrm{BC}\end{array}$ & $\begin{array}{c}8,64 \mathrm{a} \\
\mathrm{C}\end{array}$ & $\begin{array}{c}7,65 \mathrm{a} \\
\mathrm{C}\end{array}$ & $\begin{array}{c}12,26 \mathrm{a} \\
\mathrm{AB}\end{array}$ & $\begin{array}{c}14,09 \mathrm{ab} \\
\mathrm{A}\end{array}$ & $\begin{array}{c}9,94 \mathrm{a} \\
\mathrm{BC}\end{array}$ & $\begin{array}{c}8,95 \mathrm{a} \\
\mathrm{BC}\end{array}$ & $\begin{array}{c}8,91 \mathrm{~b} \\
\mathrm{BC}\end{array}$ & $9,77 \mathrm{a}$ \\
\hline diclosulam & 35 & $\begin{array}{l}\text { 10,11 a } \\
\text { BCDE }\end{array}$ & $\begin{array}{c}8,54 \mathrm{a} \\
\mathrm{DE}\end{array}$ & $\begin{array}{c}6,91 \mathrm{a} \\
\mathrm{E}\end{array}$ & $\begin{array}{l}11,62 \mathrm{a} \\
\mathrm{ABCD}\end{array}$ & $\begin{array}{c}12,61 \mathrm{a} \\
\mathrm{AB}\end{array}$ & $\begin{array}{l}9,59 \mathrm{a} \\
\text { BCDE }\end{array}$ & $\begin{array}{l}9,03 \mathrm{a} \\
\mathrm{CDE}\end{array}$ & $\begin{array}{c}14,21 \mathrm{a} \\
\mathrm{A}\end{array}$ & $\begin{array}{c}12,16 \mathrm{~b} \\
\mathrm{ABC}\end{array}$ & $\begin{array}{l}9,62 \mathrm{a} \\
\mathrm{BCDE}\end{array}$ & $\begin{array}{c}8,80 \mathrm{a} \\
\mathrm{CDE}\end{array}$ & $\begin{array}{c}10,08 \mathrm{ab} \\
\mathrm{BCDE}\end{array}$ & $10,27 \mathrm{a}$ \\
\hline diclosulam & 70 & $\begin{array}{c}9,47 \mathrm{a} \\
\mathrm{DE}\end{array}$ & $\begin{array}{l}9,62 \mathrm{a} \\
\mathrm{CDE}\end{array}$ & $\begin{array}{c}7,03 \mathrm{a} \\
\mathrm{E}\end{array}$ & $\begin{array}{c}13,00 \mathrm{a} \\
\mathrm{ABC}\end{array}$ & $\begin{array}{c}11,28 \mathrm{a} \\
\mathrm{BCD}\end{array}$ & $\begin{array}{c}8,30 \mathrm{a} \\
\mathrm{DE}\end{array}$ & $\begin{array}{c}8,92 \mathrm{a} \\
\mathrm{DE}\end{array}$ & $\begin{array}{c}13,11 \mathrm{a} \\
\mathrm{AB}\end{array}$ & $\begin{array}{c}15,58 \mathrm{a} \\
\mathrm{A}\end{array}$ & $\begin{array}{l}10,06 \text { a } \\
\text { BCDE }\end{array}$ & $\begin{array}{c}10,98 \mathrm{a} \\
\mathrm{BCD}\end{array}$ & $\begin{array}{c}9,63 \mathrm{ab} \\
\mathrm{CDE}\end{array}$ & $10,58 \mathrm{a}$ \\
\hline flumetsulam & 120 & $\begin{array}{c}8,27 \mathrm{a} \\
\mathrm{BC}\end{array}$ & $\begin{array}{c}9,58 \mathrm{a} \\
\mathrm{BC}\end{array}$ & $\begin{array}{c}8,72 \mathrm{a} \\
\mathrm{BC}\end{array}$ & $\begin{array}{c}11,29 \mathrm{a} \\
\mathrm{AB}\end{array}$ & $\begin{array}{c}10,09 \text { a } \\
\text { BC }\end{array}$ & $\begin{array}{c}8,32 \mathrm{a} \\
\mathrm{BC}\end{array}$ & $\begin{array}{c}7,08 \mathrm{a} \\
\mathrm{C}\end{array}$ & $\begin{array}{c}11,53 \mathrm{a} \\
\mathrm{AB}\end{array}$ & $\begin{array}{c}14,71 \mathrm{ab} \\
\mathrm{A}\end{array}$ & $\begin{array}{c}11,00 \mathrm{a} \\
\mathrm{B}\end{array}$ & $\begin{array}{c}9,69 \mathrm{a} \\
\mathrm{BC}\end{array}$ & $\begin{array}{c}10,51 \mathrm{ab} \\
\mathrm{B}\end{array}$ & $10,07 \mathrm{a}$ \\
\hline flumetsulam & 240 & $\begin{array}{l}8,57 \mathrm{a} \\
\mathrm{CDE}\end{array}$ & $\begin{array}{c}8,32 \mathrm{a} \\
\mathrm{DE}\end{array}$ & $\begin{array}{c}7,30 \mathrm{a} \\
\mathrm{E}\end{array}$ & $\begin{array}{c}11,92 \mathrm{a} \\
\mathrm{ABC}\end{array}$ & $\begin{array}{c}11,27 \mathrm{a} \\
\mathrm{BCD}\end{array}$ & $\begin{array}{l}9,39 \mathrm{a} \\
\mathrm{CDE}\end{array}$ & $\begin{array}{c}8,44 \mathrm{a} \\
\mathrm{DE}\end{array}$ & $\begin{array}{c}13,59 \mathrm{a} \\
\mathrm{AB}\end{array}$ & $\begin{array}{c}15,18 \mathrm{ab} \\
\mathrm{A}\end{array}$ & $\begin{array}{c}10,80 \mathrm{a} \\
\mathrm{BCD}\end{array}$ & $\begin{array}{c}8,98 \mathrm{a} \\
\mathrm{CDE}\end{array}$ & $\begin{array}{c}9,26 \mathrm{ab} \\
\mathrm{CDE}\end{array}$ & $10,25 \mathrm{a}$ \\
\hline $\begin{array}{l}\text { diclosulam + } \\
\text { flumetsulam }\end{array}$ & $20+100$ & $\begin{array}{c}8,46 \text { a } \\
\mathrm{CD}\end{array}$ & $\begin{array}{c}9,66 \mathrm{a} \\
\mathrm{BCD}\end{array}$ & $\begin{array}{c}7,94 \mathrm{a} \\
\mathrm{D}\end{array}$ & $\begin{array}{c}11,49 \mathrm{a} \\
\mathrm{ABC}\end{array}$ & $\begin{array}{c}11,65 \mathrm{a} \\
\mathrm{ABC}\end{array}$ & $\begin{array}{c}8,98 \mathrm{a} \\
\mathrm{BCD}\end{array}$ & $\begin{array}{c}8,31 \mathrm{a} \\
\mathrm{CD}\end{array}$ & $\begin{array}{c}11,55 \mathrm{a} \\
\mathrm{ABC}\end{array}$ & $\begin{array}{c}14,71 \mathrm{ab} \\
\mathrm{A}\end{array}$ & $\begin{array}{c}10,38 \mathrm{a} \\
\mathrm{BCD}\end{array}$ & $\begin{array}{c}9,66 \text { a } \\
\text { BCD }\end{array}$ & $\begin{array}{c}12,37 \mathrm{a} \\
\mathrm{AB}\end{array}$ & $10,43 \mathrm{a}$ \\
\hline imazaquin & 150 & $\begin{array}{c}6,84 \mathrm{a} \\
\mathrm{E}\end{array}$ & $\begin{array}{c}10,31 \mathrm{a} \\
\mathrm{CD}\end{array}$ & $\begin{array}{c}7,57 \mathrm{a} \\
\mathrm{DE}\end{array}$ & $\begin{array}{c}12,45 \mathrm{a} \\
\mathrm{ABC}\end{array}$ & $\begin{array}{c}10,98 \text { a } \\
\text { BCD }\end{array}$ & $\begin{array}{c}10,11 \mathrm{a} \\
\mathrm{CDE}\end{array}$ & $\begin{array}{c}8,74 \mathrm{a} \\
\mathrm{DE}\end{array}$ & $\begin{array}{c}13,83 \mathrm{a} \\
\mathrm{AB}\end{array}$ & $\begin{array}{c}15,52 \mathrm{a} \\
\mathrm{A}\end{array}$ & $\begin{array}{c}10,52 \mathrm{a} \\
\mathrm{BCD}\end{array}$ & $\begin{array}{l}9,73 \mathrm{a} \\
\mathrm{CDE}\end{array}$ & $\begin{array}{c}10,68 \mathrm{ab} \\
\mathrm{BCD}\end{array}$ & $10,61 \mathrm{a}$ \\
\hline $\begin{array}{l}\text { MÉDIA G } \\
\text { CULTIV }\end{array}$ & $\begin{array}{l}\text { ERAL } \\
\text { IRES }\end{array}$ & $\begin{array}{l}8,53 \\
\text { FGH }\end{array}$ & $\begin{array}{l}9,39 \\
\text { EFG }\end{array}$ & $\begin{array}{c}7,56 \\
\mathrm{H}\end{array}$ & $\begin{array}{c}11,99 \\
\mathrm{BC}\end{array}$ & $\begin{array}{c}11,06 \\
\mathrm{CD}\end{array}$ & $\begin{array}{l}9,05 \\
\text { EFG }\end{array}$ & $\begin{array}{l}8,31 \\
\mathrm{GH}\end{array}$ & $\begin{array}{c}12,87 \\
\text { B }\end{array}$ & $\begin{array}{c}14,57 \\
\mathrm{~A}\end{array}$ & $\begin{array}{c}10,34 \\
\mathrm{DE}\end{array}$ & $\begin{array}{c}9,54 \\
\mathrm{EF}\end{array}$ & $\begin{array}{c}10,21 \\
D E\end{array}$ & -- \\
\hline
\end{tabular}

* Médias seguidas pela mesma letra não diferem entre si pelo Teste de Tukey ao nível de 5\% de probabilidade.

** Letras minúsculas a direita de cada valor, representam as médias dos tratamentos dentro de cada variedade e devem ser comparadas na vertical.

*** Letras maiúsculas sob cada valor, correspondem as médias de cultivares dentro de cada tratamento e devem ser comparadas na horizontal.

**** Dados originais foram transformados em $\mathrm{y}^{0,317}$. 
TABELA 10. Rendimentos de grãos ( $\mathrm{Kg} / \mathrm{ha}$ ) de cultivares de soja de diferentes ciclos sob efeito de herbicidas, aplicados em pré-emergência. Londrina - PR, 1998.

\begin{tabular}{|c|c|c|c|c|c|c|c|c|c|c|c|c|c|c|}
\hline \multirow{2}{*}{\multicolumn{2}{|c|}{ TRATAMENTOS }} & \multicolumn{12}{|c|}{ CULTIVARES } & \multirow{3}{*}{$\begin{array}{c}\text { MÉDIA } \\
\text { GERAL } \\
\text { HERBICIDA }\end{array}$} \\
\hline & & \multicolumn{3}{|c|}{ PRECOCE } & \multicolumn{4}{|c|}{ SEMIPRECOCE } & \multicolumn{5}{|c|}{ MÉDIO } & \\
\hline HERBICIDA & $\begin{array}{l}\text { DOSE } \\
\text { g i.a/ha }\end{array}$ & $\begin{array}{c}\text { FT- } \\
\text { GUAIRA }\end{array}$ & $\begin{array}{l}\text { EMBRAPA- } \\
48 \\
\end{array}$ & $\begin{array}{c}\text { OCEPAR } \\
14 \\
\end{array}$ & BR-16 & $\begin{array}{c}\text { OCEPAR } \\
13 \\
\end{array}$ & $\begin{array}{l}\text { EMBRAPA- } \\
\quad 4 \\
\end{array}$ & BR-36 & $\begin{array}{c}\text { FT-10 } \\
\text { PRINCESA }\end{array}$ & $\begin{array}{c}\text { FT } \\
2000\end{array}$ & $\begin{array}{c}\text { FT } \\
\text { ABYARA }\end{array}$ & BR-37 & $\begin{array}{c}\text { RS-7 } \\
\text { JACUI }\end{array}$ & \\
\hline $\begin{array}{c}\text { Testemunha } \\
\text { capinada }\end{array}$ & -- & $\begin{array}{c}3.426 \mathrm{a} \\
\mathrm{A}\end{array}$ & $\begin{array}{c}3.480 \mathrm{a} \\
\mathrm{A}\end{array}$ & $\begin{array}{c}3.463 \mathrm{a} \\
\mathrm{A}\end{array}$ & $\begin{array}{c}2.843 \mathrm{a} \\
\mathrm{A}\end{array}$ & $\begin{array}{c}2.808 \mathrm{a} \\
\mathrm{A}\end{array}$ & $\begin{array}{c}3.328 \mathrm{a} \\
\mathrm{A}\end{array}$ & $\begin{array}{c}3.264 \mathrm{a} \\
\mathrm{A}\end{array}$ & $\begin{array}{c}3.134 \mathrm{a} \\
\mathrm{A}\end{array}$ & $\begin{array}{c}2.748 \mathrm{a} \\
\mathrm{A}\end{array}$ & $\begin{array}{c}2.675 \mathrm{a} \\
\mathrm{A}\end{array}$ & $\begin{array}{c}3.373 \mathrm{a} \\
\mathrm{A}\end{array}$ & $\begin{array}{c}2.731 \mathrm{a} \\
\mathrm{A}\end{array}$ & $3.106 \mathrm{~b}$ \\
\hline diclosulam & 35 & $\begin{array}{c}3.350 \mathrm{a} \\
\mathrm{AB}\end{array}$ & $\begin{array}{c}3.716 \mathrm{a} \\
\mathrm{A}\end{array}$ & $\begin{array}{c}3.510 \mathrm{a} \\
\mathrm{AB}\end{array}$ & $\begin{array}{c}3.404 \mathrm{a} \\
\mathrm{AB}\end{array}$ & $\begin{array}{c}3.066 \mathrm{a} \\
\mathrm{AB}\end{array}$ & $\begin{array}{c}3.406 \mathrm{a} \\
\mathrm{AB}\end{array}$ & $\begin{array}{c}3.326 \mathrm{a} \\
\mathrm{AB}\end{array}$ & $\begin{array}{c}3.462 \mathrm{a} \\
\mathrm{AB}\end{array}$ & $\begin{array}{c}2.757 \mathrm{a} \\
\mathrm{AB}\end{array}$ & $\begin{array}{c}3.004 \mathrm{a} \\
\mathrm{AB}\end{array}$ & $\begin{array}{c}3.195 \mathrm{a} \\
\mathrm{AB}\end{array}$ & $\begin{array}{c}2.539 \mathrm{a} \\
\mathrm{B}\end{array}$ & $3.228 \mathrm{ab}$ \\
\hline diclosulam & 70 & $\begin{array}{c}2.709 \mathrm{a} \\
\mathrm{A} \\
\end{array}$ & $\begin{array}{c}3.659 \text { a } \\
\text { A } \\
\end{array}$ & $\begin{array}{c}3.351 \mathrm{a} \\
\mathrm{A} \\
\end{array}$ & $\begin{array}{c}2.850 \mathrm{a} \\
\mathrm{A} \\
\end{array}$ & $\begin{array}{c}3.347 \mathrm{a} \\
\mathrm{A} \\
\end{array}$ & $\begin{array}{c}2.933 \mathrm{a} \\
\mathrm{A} \\
\end{array}$ & $\begin{array}{c}3.403 \mathrm{a} \\
\mathrm{A} \\
\end{array}$ & $\begin{array}{c}3.621 \mathrm{a} \\
\mathrm{A} \\
\end{array}$ & $\begin{array}{c}2.994 \mathrm{a} \\
\mathrm{A} \\
\end{array}$ & $\begin{array}{c}3.078 \mathrm{a} \\
\mathrm{A} \\
\end{array}$ & $\begin{array}{c}2.999 \text { a } \\
\text { A } \\
\end{array}$ & $\begin{array}{c}2.771 \mathrm{a} \\
\mathrm{A} \\
\end{array}$ & $3.143 \mathrm{ab}$ \\
\hline flumetsulam & 120 & $\begin{array}{c}3.253 \mathrm{a} \\
\mathrm{A}\end{array}$ & $\begin{array}{c}3.654 \mathrm{a} \\
\mathrm{A}\end{array}$ & $\begin{array}{c}3.561 \mathrm{a} \\
\mathrm{A}\end{array}$ & $\begin{array}{c}2.989 \mathrm{a} \\
\mathrm{A}\end{array}$ & $\begin{array}{c}2.724 \mathrm{a} \\
\mathrm{A}\end{array}$ & $\begin{array}{c}3.434 \mathrm{a} \\
\mathrm{A}\end{array}$ & $\begin{array}{c}3.340 \mathrm{a} \\
\mathrm{A}\end{array}$ & $\begin{array}{c}3.135 \mathrm{a} \\
\mathrm{A}\end{array}$ & $\begin{array}{c}2.777 \mathrm{a} \\
\mathrm{A}\end{array}$ & $\begin{array}{c}3.270 \mathrm{a} \\
\mathrm{A}\end{array}$ & $\begin{array}{c}3.409 \mathrm{a} \\
\mathrm{A}\end{array}$ & $\begin{array}{c}3.019 \mathrm{a} \\
\mathrm{A}\end{array}$ & $3.214 \mathrm{ab}$ \\
\hline flumetsulam & 240 & $\begin{array}{c}3.184 \mathrm{a} \\
\mathrm{A}\end{array}$ & $\begin{array}{c}3.551 \mathrm{a} \\
\mathrm{A}\end{array}$ & $\begin{array}{c}3.194 \mathrm{a} \\
\mathrm{A}\end{array}$ & $\begin{array}{c}3.643 \mathrm{a} \\
\mathrm{A}\end{array}$ & $\begin{array}{c}2.993 \mathrm{a} \\
\mathrm{A}\end{array}$ & $\begin{array}{c}3.224 \mathrm{a} \\
\mathrm{A}\end{array}$ & $\begin{array}{c}3.335 \mathrm{a} \\
\mathrm{A}\end{array}$ & $\begin{array}{c}3.029 \mathrm{a} \\
\text { A }\end{array}$ & $\begin{array}{c}2.871 \mathrm{a} \\
\mathrm{A}\end{array}$ & $\begin{array}{c}2.975 \mathrm{a} \\
\mathrm{A}\end{array}$ & $\begin{array}{c}3.173 \mathrm{a} \\
\mathrm{A}\end{array}$ & $\begin{array}{c}2.860 \mathrm{a} \\
\mathrm{A}\end{array}$ & $3.169 \mathrm{ab}$ \\
\hline $\begin{array}{l}\text { diclosulam + } \\
\text { flumetsulam }\end{array}$ & $20+100$ & $\begin{array}{c}3.278 \mathrm{a} \\
\mathrm{A}\end{array}$ & $\begin{array}{c}3.650 \mathrm{a} \\
\mathrm{A}\end{array}$ & $\begin{array}{c}3.218 \mathrm{a} \\
\mathrm{A}\end{array}$ & $\begin{array}{c}3.431 \mathrm{a} \\
\mathrm{A}\end{array}$ & $\begin{array}{c}3.292 \mathrm{a} \\
\text { A }\end{array}$ & $\begin{array}{c}3.156 \mathrm{a} \\
\mathrm{A}\end{array}$ & $\begin{array}{c}3.621 \mathrm{a} \\
\mathrm{A}\end{array}$ & $\begin{array}{c}3.401 \mathrm{a} \\
\mathrm{A}\end{array}$ & $\begin{array}{c}3.045 \mathrm{a} \\
\mathrm{A}\end{array}$ & $\begin{array}{c}3.086 \mathrm{a} \\
\mathrm{A}\end{array}$ & $\begin{array}{c}3.162 \mathrm{a} \\
\mathrm{A}\end{array}$ & $\begin{array}{c}3.099 \mathrm{a} \\
\text { A }\end{array}$ & $3.287 \mathrm{ab}$ \\
\hline imazaquin & 150 & $\begin{array}{c}3.219 \mathrm{a} \\
\mathrm{A} \\
\end{array}$ & $\begin{array}{c}3.844 \mathrm{a} \\
\mathrm{A} \\
\end{array}$ & $\begin{array}{c}3.523 \mathrm{a} \\
\mathrm{A} \\
\end{array}$ & $\begin{array}{c}3.171 \mathrm{a} \\
\mathrm{A}\end{array}$ & $\begin{array}{c}2.928 \mathrm{a} \\
\mathrm{A} \\
\end{array}$ & $\begin{array}{c}3.326 \mathrm{a} \\
\mathrm{A} \\
\end{array}$ & $\begin{array}{c}3.503 \mathrm{a} \\
\mathrm{A} \\
\end{array}$ & $\begin{array}{c}3.624 \mathrm{a} \\
\mathrm{A} \\
\end{array}$ & $\begin{array}{c}3.484 \mathrm{a} \\
\mathrm{A} \\
\end{array}$ & $\begin{array}{c}3.090 \mathrm{a} \\
\mathrm{A} \\
\end{array}$ & $\begin{array}{c}3.700 \mathrm{a} \\
\mathrm{A} \\
\end{array}$ & $\begin{array}{c}3.007 \mathrm{a} \\
\mathrm{A} \\
\end{array}$ & $3.368 \mathrm{a}$ \\
\hline $\begin{array}{l}\text { MÉDIA G } \\
\text { CULTIV }\end{array}$ & $\begin{array}{l}\text { ERAL } \\
\text { ARES }\end{array}$ & $\begin{array}{c}3.203 \\
\text { A }\end{array}$ & $\begin{array}{c}3.651 \\
\mathrm{~A}\end{array}$ & $\begin{array}{c}3.403 \\
\mathrm{~A}\end{array}$ & $\begin{array}{c}3.190 \\
\text { A }\end{array}$ & $\begin{array}{c}3.023 \\
\mathrm{~A}\end{array}$ & $\begin{array}{c}3.258 \\
\mathrm{~A}\end{array}$ & $\begin{array}{c}3.399 \\
\text { A }\end{array}$ & $\begin{array}{c}3.344 \\
\text { A }\end{array}$ & $\begin{array}{c}2.954 \\
\text { A }\end{array}$ & $\begin{array}{c}3.025 \\
\mathrm{~A}\end{array}$ & $\begin{array}{c}3.287 \\
\mathrm{~A}\end{array}$ & $\begin{array}{c}2.861 \\
\mathrm{~A}\end{array}$ & -- \\
\hline
\end{tabular}

* Médias seguidas pela mesma letra não diferem entre si pelo Teste de Tukey ao nível de 5\% de probabilidade.

** Letras minúsculas a direita de cada valor, representam as médias dos tratamentos dentro de cada variedade e devem ser comparadas na vertical.

*** Letras maiúsculas sob cada valor, correspondem as médias de cultivares dentro de cada tratamento e devem ser comparadas na horizontal. 
No entanto, o chloransulam-methyl, outro componente do grupo, tem o seu mecanismo de seletividade devido a conjugação com homoglutationa (Hess \& Anderson, 1995). Portanto, especula-se, por analogia, que os caminhos potenciais de detoxificação do diclosulam em soja podem ser a hidroxilação seguida de conjugação com glicose e/ou conjugação com homoglutationa. Então, espera-se que cultivares de soja com semelhantes mecanismos podem tolerar melhor estes herbicidas.

Considerando ainda o comportamento desses herbicidas no solo, que em função de determinadas condições ou fatores, tem sua atividade melhorada ou não, podemos entender melhor a tolerância relativa. Esta poderá ser maior naquelas cultivares de ciclo mais longo, e menor para aquelas de ciclo precoce, pois as primeiras terão um tempo maior para recuperação no caso de um estresse químico. Devido a natureza ácidofraco do flumetsulam, sua adsorção aos coloídes do solo é fortemente dependente do $\mathrm{pH}$ do meio, além dos teores de argila e matéria orgânica (Fontaine, 1991). Por causa de seu pKa (constante de dissociação) de 4,6 o composto existe nas formas neutras e aniônicas na maioria dos solos agrícolas, com mais alta proporção de ânions em pH mais elevado. Dessa forma a constante de adsorção (Kd) deste herbicida é a somatória das constantes de adsorção da forma neutra e aniônica. Este mesmo autor estudando a adsorção do flumetsulam em 21 tipos de solos dos USA encontrou valores de $\mathrm{K}_{\mathrm{oc}}$ (valores de $\mathrm{Kd}$ dividido pelo teor de carbono orgânico) de $12 \pm 1 \mathrm{ml} / \mathrm{g}$ de solo da forma aniônica e $650 \pm 40 \mathrm{ml} / \mathrm{g}$ da forma neutra. Em experimento adicional foi ajustado níveis de $\mathrm{pH}$ em 4 solos e foi encontrado na média $750 \mathrm{ml} / \mathrm{g}$ de solo para a forma neutra. Ou seja, ocorre um aumento acentuado da adsorção com a diminuição do $\mathrm{pH}$. Isto indica ainda uma menor mobilidade da forma neutra $\left(\mathrm{K}_{\mathrm{oc}}\right.$ mais alto), ao passo que a forma aniônica ( $\mathrm{K}_{\mathrm{oc}}$ mais baixos) possue uma maior mobilidade na solução do solo. Baseado nestes dados o autor afirma que isto pode ser aplicado a outros compostos com valores de
pKa na faixa de $\mathrm{pH}$ do solo, como por exemplo o diclosulam. A adsorção dependente do $\mathrm{pH}$ para outros herbicidas ácidos foram relatadas por Walker et al. (1989), Stougaard et al. (1990), no caso das imidazolinonas, e no caso do pentaclorofenol Lee et al. (1990), citados por Fontaine (1991), descrevem que a adsorção do pentaclorofenol é dependente das proporções da forma neutra e aniônica.

Foi observado que a meia-vida do flumetsulam é menor em solos com menor capacidade de adsorção. Esse valor é de um mês ou menos em solos com pH igual ou superior a 7,0 e matéria orgânica menor que $4,0 \%$, ou em $\mathrm{pH}$ entre 6,0 e 7,0 e menos de $1,0 \%$ de matéria orgânica; e de 1-4 meses em solo com pH entre 6,0 e 7,0 e matéria orgânica entre 2,0 e 4,0\% (Lehmann et al., 1992). Esta última situação é a mais parecida com o presente experimento, no qual o $\mathrm{pH}$ foi menor $(5,4)$, sugerindo, neste caso, uma maior persistência dos herbicidas. Há indicações de uma maior degradação do flumetsulam quando presente na solução do solo, e ao que parece principalmente por via microbiana, conforme estes mesmos autores. No caso do diclosulam, em relação a adsorção, não foi encontrado nada na literatura, mas por analogia, sabendo-se ser também um ácido-fraco, espera-se comportamento semelhante ao flumetsulam. Porém, devido ao seu pKa ser um pouco mais baixo $(4,2)$ e ter uma menor solubilidade, deverá apresentar uma menor mobilidade, principalmente em $\mathrm{pH}$ mais baixos, e uma maior persistência no solo.

Portanto, baseado no que foi exposto, nas condições do presente experimento, solo com $\mathrm{pH}$ 5,4 , argila $80,84 \%$ e $3,07 \%$ de matéria orgânica, há indicativos, principalmente em função do $\mathrm{pH}$ relativamente baixo, de que existiu um certo equilíbrio das duas formas iônicas, com predominância da aniônica para os dois herbicidas. Desta maneira uma grande porcentagem de moléculas adsorvidas pode ter sido decisivas para que doses letais não atingissem o sítio de ação, isto somado a possível 
maior velocidade de degradação da porção aniônica presente na solução do solo, em razão de altas temperaturas e constante umidade no decorrer do experimento. Tudo isto pode ter contribuído para a não expressão de injúria às plantas de soja, principalmente com as doses mais elevadas com as quais esperava-se, pelo menos o aparecimento dos sintomas de injúria.

Pode-se concluir, que em doses normais recomendadas, os herbicidas flumetsulam ( $120 \mathrm{~g} / \mathrm{ha})$ e diclosulam (35 g/ha) são seguros para as 12 cultivares de soja estudadas, em condições de solo e clima semelhantes às que foram avaliadas. Porém, na eventualidade de ocorrência de doses dobradas, o que é comum nas sobreposições de faixa de aplicação, o potencial de risco de fitotoxicação existe, principalmente naquelas cultivares com indicativos de sensibilidade, ou seja, 'Embrapa-4', 'FT-Guaira' e 'BR-37', e sobretudo, em solos arenosos com pH elevado e baixo teor de matéria orgânica.

\section{LITERATURA CITADA}

BELTRÃO, N.E.M., SILVA, J.F.; SILVEIRA, A.J., SEDIYAMA. C.S., COSTA, L.M., OLIVA, M.A. Resistência de espécies e cultivares de algodão (Gossipium spp.) ao herbicida diuron. Planta Daninha, v. 6, n.1, p. 72-78, 1983.

BOX, G.E.P., HUNTER,W.G., HUNTER,J.S. Statistics for Experiments. New York, John Wiley. . p.231-241,1978.

BRONHARA, A. A. \& MENGARDA, I. P. Estudo da atividade do herbicida flumetsulam para o controle de plantas daninhas dicotiledôneas infestantes da cultura da soja no sul do Brasil. Sumário de dados experimentais, sul do Brasil entre 1985-90. In.: Congresso Brasileiro de Herbicidas e Plantas Daninhas, $19^{\circ}$, Londrina, Resumos p. 88-89, 1993.
BRONHARA, A.A. \& MENGARDA, I.P. Estudo da atividade do herbicida flumetsulam para o controle de plantas daninhas dicotiledôneas infestantes da cultura de soja no centro oeste brasileiro. Sumário de dados experimentais, centro oeste brasileiro entre 1985-90. In.: Congresso Brasileiro de Herbicidas e Plantas Daninhas, $19^{\circ}$, Londrina, Resumos p. 116-117, 1993.

BUZZELL, R.I. \& HAMILL A.S. Improved Tolerance of Soybean (Glycine max) to metribuzin. Weed Technol., v.2, n. 2, p. 170-171, 1988.

CUNHA, R. A. , RODRIGUES, J.J.V. , SEDIYAMA, C. S., COSTA, L.M., SILVA, J.F. Tolerância de cultivares de soja (Glycine max (L.)Merryl) ao diuron e metribuzin em diferentes tipos de solos. In.: Congresso Brasileiro de Herbicidas de Plantas Daninhas, $15^{\circ}$, Belo Horizonte, Resumos p. 100-101, 1984.

CUNHA, R. A. , RODRIGUES, J.J.V., SEDIYAMA, C. S., COSTA, L.M., SILVA, J.F. Tolerância de cultivares de soja (Glycine max (L.) Merryl) ao diuron e metribuzin em diferentes níveis de matéria orgânica no solo. In.: Congresso Brasileiro de Herbicidas de Plantas Daninhas, $15^{\circ}$, Belo Horizonte, Resumos p. 101-102, 1984.

DAYAN, F.E. , WEETE, J.D. , DUKE, S.O. , HANCOCK, H.G. Soybean (Glycine max) cultivar differences in response to sulfentrazone. Weed Sci., v.45, p. 634-641, 1997.

EBERLEIN, C.V., ROSOW, K.M., GEADELMANN, J.L., OPENSHAW, S.J. Differential tolerance of corn genotypes to DPX-M6316. Weed Sci., v.37, n. 5 p. 651$657,1989$. 
EDWARDS, C.J., BARRENTINE, W.L., KILEN, T.C. Inheritance of sensitivity to metribuzin in soybeans. Crop Sci., v. 16, p.119-120, 1976.

FALB, L. N. \& SMITH, A. E. Metribuzin metabolism in soybeans: Characterization of the intraspecific differential tolerance. $\mathbf{J}$. Agric. Food Chem., v. 32, p. 1425, 1984.

FONTAINE, D.D. , LEHMANN, R.G. , MILLER, J.R. Soil adsorption of neutral and anionic forms of a sulfonamide herbicide, flumetsulam. J. Environ. Qual., v. 20, p. 759-762, 1991.

FRIESEN, G.H. \& WALL, D.A. Tolerance of early-maturing soybean cultivars to metribuzin. Can. J. Plant. Sci., v. 66, p.125-130,1986.

GAZZIERO, D.L.P.; MENOSSO, O.G. Comportamento de 24 cultivares de soja recomendada para o estado do Paraná ao herbicida metribuzin. In.: Congresso Brasileiro de Herbicidas de Plantas Daninhas, $15^{\circ}$, Belo Horizonte, Resumo p. 99, 1984.

GERWICK, B.C. , SUBRAMANIAN, M.V. , LONEY-GALLANT, V.I. , CHANDLER, D.P. Mechanism of Action of the 1,2,4Triazolo [ 1,5-a] pyrimidines. Pestic. Sci., v. 29, n. 3, p.357-364, 1990.

GUIMARÃES, S.C. \& VALENTE, T.O. Avaliação do flumetsulam em soja no Mato Grosso. In.: Congresso Brasileiro da Ciência das Plantas Daninhas, 20, Florianópolis, Resumos, p. 72-73, 1995.

HESS, F.D. \& ANDERSON,R. Herbicides in development in the U.S. In.: Herbicide action. West Lafayette: Purdue University, Indiana, p.407, 1995.
HODGES, C.C. , DE BOER, G.J. , AVALOS, J. Uptake and Metabolism as Mechanism of Selective Herbicidae Activity of the 1,2, 4 - Triazolo [ 1, 5 - a] pyrimidines. Pestic. Sci., v. 29, n. 3, p.365-378, 1990.

KLESCHICK, W.A., COSTALES, M.J., DUNBAR, J.E., MEIKLE, R.W., MONTE, W.T., PEARSON, N.R., SNIDER, S.W., VINOGRADOFF, A.P. New Herbicidal Derivatives of 1,2,4-Triazolo [1,5-a] pyrimidine. Pestic. Sci., v. 29, n. 3, p.341355, 1990.

KLESCHICK, W.A., GERWICK, B.C., CARSON, C.M. , MONTE, W.T., SNIDER, S.W. DE 498, a new acetolactate synthase inhiting herbicide with multicrop. selectivity. J. Agric. Food. Chem. v. 40, n. 6, p. 1083-1085, 1992.

KRUSE, N.D. , RUBIN, R. , BAVARESCO, A. Avaliação da eficiência agronômica e seletividade do herbicida diclosulan, aplicado em pré-plantio incorporado, no controle de Euphorbia heterophylla, na cultura da soja. In.: Congresso Brasileiro da Ciência das Plantas Daninhas, 21 ${ }^{\circ}$, Caxambu, Resumos, p. 99, 1997.

LEHMANN, R.G., MILLER, J.R., FONTAINE, D.D., LASKOSWSK, D.A., HUNTER,J.H., CORDES, R.C. Degradation of a sulfonamide as a funcion of soil sortion. Weed Res., v. 32, p. 197-205, 1992.

MAGALHÃES, P.M. \& BARROS, A.C de. Eficiência e seletividade do herbicida flumetsulam no controle de invasoras dicotiledôneas na cultura da soja. In.: Congresso Brasileiro de Herbicidas e Plantas Daninhas, $19^{\circ}$, Londrina, Resumos, p. 118-119, 1993. 
MANGEOT, B.L., SLIFE, F.E., RIECK, C.E. Differential metabolism of metribuzin by two soybean (Glycine max) cultivars. Weed Sci., v.27, p. 267-269, 1979.

MARTIN, M.D., WORTHINGTON, J.P., GRAY, E. Soybean (Glycine max) Cultivar Response to Fluchloralin, Metribuzin, and Vernolate. Weed Technol., v. 1, n. 4, p.282-285, 1987.

NEWSOM, L.J. \& SHAW, D.R. Soybean (Glycine max) cultivar tolerance to chlorimuron and imazaquin with varying hydroponic solution $\mathrm{pH}$. Weed Technol., v. 6, n. 2, p. 382-388, 1992.

PELISSARI, A. , RONZELLI Jr., P. , KOEHLER, H.S. Estudo da eficiência agronômica de herbicidas do grupo químico das sulfonamidas aplicados em pré-plantio incorporado e pós-emergência na cultura da soja (Glycine max) cultivar FT Guaira. In.: Congresso Brasileiro da Ciência das Plantas Daninhas, $21^{\circ}$, Caxambu, Resumos, p. 118, 1997.

PEREIRA, R.C., AMABILE, R.F., SOUZA, P.I.M. Tolerância diferencial de cultivares de soja aos herbicidas cyanazine e metribuzin. In.: Congresso Brasileiro de Herbicidas e Plantas Daninhas, $17^{\circ}$, Piracicaba, Resumos p. 109-110, 1988.

ROCHA, M.A.L. Avaliação da eficiência do herbicida flumetsulam aplicado em préplantio incorporado no controle de plantas daninhas de folhas largas na cultura da soja. In.: Congresso Brasileiro de Herbicidas e Plantas Daninhas, $19^{\circ}$, Londrina, Resumos p. 117-118, 1993.
SANDER, K.W. \& BARRETT, M. Differential imazaquin tolerance and behavior in selected corn (Zea mays) hybrids. Weed Sci., v. 37, n. 3, p.290-295, 1989.

STOUGAARD, R.N. , SHEA, P.J. , MARTIN, A.R. Effect of soil type and $\mathrm{pH}$ on adsorption, mobility, and efficacy of imazaquin and imazethapyr. Weed Sci., v. 38, p. 67-73, 1990.

WALKER, A, COTTERILL,E,G., WELCH,S.J. Adsortion and degradation of chorsulfuron and metsulfuron-methyl in soils from different depths. Weed Res., v. 29 p. 281287, 1989.

WAX, L.M., STOLLER, E.W., BERNARD,R.L. Differencial response of soybeans cultivars to metribuzin. Agron. J., v. 68, p.484-486, 1976.

WEESE, W.W., WAX, L.M., CARLSON, W.C., CHARLETTA, J.A. Response of soybean (Glycine max) cultivars to metribuzin in the field and greenhouse. Weed Technol., v. 3, n. 4, p. 566-572, 1989.

WIXSON, M.B. \& SHAW, D.R. Differencial response of soybean (Glycine max) cultivars to AC 263,222. Weed Technol., v. 5, n. 2, p. 430-433, 1991.

ZAGONEL, J. , VENANCIO, W.S. , MILLEO, M.V.R. Controle pré-emergente de plantas daninhas na cultura da soja através do diclosulan. In.: Congresso Brasileiro da Ciência das Plantas Daninhas, 21 ${ }^{\circ}$, Caxambu, Resumos, p. 145, 1997. 